\title{
LA MONEDA VISIGODA: ANEXO I
}

\section{THE VISIGOTH COINS: ANNEX I}

\author{
RUTH PLIEGO*
}

\begin{abstract}
Resumen: Se presenta el primer Anexo al corpus de La moneda visigoda, incluyendo no solo los nuevos hallazgos que han tenido lugar en los últimos años, entre los cuales destaca el tesoro de La Vega Baja de Toledo, sino también aquellas novedades que no aparecen registradas en dicho corpus. A este grupo se añaden algunos repertorios que no pudieron ser incluidos en su momento, como sucede con el Museo de Arte Romano de Mérida, el $\mathrm{Mu}$ sée de Narbonne y el Musée du Biterrois de Béziers, entre otros. Palabras clave: Numismática, Visigodos, Monarquía visigoda, Monedas visigodas, Reino visigodo de Toledo, Corpus numismático, Anexo, Museo de Arte Romano de Mérida, Musée de Narbonne, Musée du Biterrois de Béziers.
\end{abstract}

Una de las características más destacadas de la moneda visigoda la constituye su singularidad como objeto histórico, que queda claramente puesto de relieve si se compara su producción monetaria con la de otros periodos más dilatados en el tiempo. El escaso siglo y medio en el que se acuñaron estas piezas proporciona un repertorio que, según la opinión generalizada, no sobrepasaría las diez mil monedas, una cifra muy reducida que sin embargo muestra una peculiaridad que añadida a la rareza de la serie, la hace más atractiva si cabe para el investigador. Nos referimos a la enorme cantidad de cuños conocidos en proporción al número de ejemplares registrados, lo que explica por sí mismo la amplitud del corpus numismático visigodo.

* Miembro del Grupo de Investigación 'De la Turdetania a la Bética' (HUM 152); Personal investigador del Proyecto de excelencia 'La construcción y evolución de las entidades étnicas en Andalucía en la Antigüedad (siglos VII a.C.- II d.C) (HUM-3482). Investigadora contratada por la Casa de Velázquez, Proyecto 'Detroit' (ANR)

\begin{abstract}
We present a first annex to the work La moneda visigoda, which includes not only the new finds that have taken place in recent years, among which is the treasure of La Vega Baja de Toledo, but also other coins that were not registered in the corpus. To this we will add some collections that we were then not able to incorporate, as those of the Museum of Roman Art in Merida, the Musée de Narbonne and Musée du Biterrois of Béziers, among others.

Key words: Numismatics, Visigoths, Visigothic Monarchy, Visigothic Coins, Visigothic Kingdom of Toledo, Numismatic Corpus, Annex, Museo de Arte Romano de Mérida, Musée de Narbonne, Musée du Biterrois de Béziers.
\end{abstract}

Esta peculiaridad hace que en la práctica se produzca una continua entrada de ejemplares nuevos al cuerpo monetario lo que requiere una constante puesta al día del mismo. En las líneas que siguen presentaremos las novedades que han tenido lugar desde la publicación de nuestra obra, hace ya dos años, titulada La moneda Visigoda (Sevilla, 2009). Dichas novedades, como se verá, pueden formar parte de hallazgos recientes, o de colecciones que, por distintos motivos, no pudieron integrar en su día nuestro corpus, si bien la principal fuente de innovaciones la proporcionan los catálogos de subastas de las principales firmas numismáticas. A pesar de que la mayoría de ellas no son más que variantes de las monedas conocidas, ya sea en lo que respecta a su tipología o a las leyendas, con carácter más relevante, debemos también añadir un nuevo taller desconocido hasta ahora para el reinado de Chintila (636-639) e incluso se ha incrementado el elenco de las cecas visigodas. Por otro lado se ha querido aprovechar para realizar 
algunas precisiones sobre determinadas piezas incluidas en nuestro trabajo de 2009 y que la perspectiva del tiempo ha variado, esperamos que hacia el lado correcto.

Aunque en los distintos apartados de este trabajo se comentarán todas estas novedades, la información completa de los ejemplares quedará recogida al final del artículo en forma de inventario, ordenada por reinados y cecas, con el fin de que pueda ser utilizada como un Anexo al Corpus. En total incluimos 157 monedas, contando con una falsificación de época y una reproducción moderna. El número y letra que antecede a la descripción se corresponde con el Corpus (Pliego 2009), mientras que el que precede a la referencia específica de la moneda se refiere al lugar que ocupa entre las conocidas ${ }^{1}$. En este sentido si la pieza en cuestión no puede ser incluida en algunas de las variantes registradas, como sucede cuando se trata de un taller nuevo, se duplica el número anterior del catálogo y se acompaña de 'A' (p. ej. Suintila de Vada: 431A; Chintila de Elvora: 496A.1); en el caso de variantes o bien se colocan a continuación de la última, lo que no supone ningún problema (p. ej. la última variante recogida bajo el número 276 es la 'd', por lo que la nueva variante será la 276 e: Sisebuto de Ispali: 276 e), o bien se inserta en el lugar donde debería de ir duplicando la letra (p. ej. Sisenando de Acci: cat. 438 gg.1) 2 .

1. Algunas de estas, sobre todo en lo que respecta al repertorio del Museo de Mérida, ya aparecen en nuestro corpus citadas a través del catálogo de subasta correspondiente. Ese es el motivo de que en algunos casos el número de las monedas citadas no sea correlativo.

2. Son muchas las personas a las que queremos mostrar nuestra gratitud por su ayuda a la hora de proporcionarnos información de los ejemplares recogidos en este trabajo. Sobre el hallazgo de La Vega Baja de Toledo, a los arqueólogos que hallaron el tesorillo de tremises visigodos, Rafael Caballero, Bienvenido Maquedano y Elena Sánchez, en particular a esta última por su diligencia en el envío de las fotografías de las monedas. Otros datos de ese sitio fueron facilitados con prontitud por Jorge de Juan Ares y Miguel Ángel Valero. Debemos mencionar también en relación con los mismos ejemplares a $\mathrm{Su}$ sana Cortes del Museo de Santa Cruz de Toledo y a su director, Alfonso Caballero. Por otro lado la información sobre el repertorio del Museo de Arte Romano de Mérida vino de la mano de su director, José María Álvarez, a través del conservador Agustín Velázquez. Por su parte la conservadora del Museo de Jaén, Margarita Sánchez Latorre nos envió la fotografía -realizada por Salvador Gómez Luquín- de la única moneda visigoda de ese museo. Asimismo mostramos nuestro agradecimiento al Director del Museo de Guadalajara, Fernando Aguado, y al conservador, Miguel Ángel Cuadrado, así como a Fernando Colodro (Fundación Cultura y Misión, Martos, Jaén). Con respecto a la colección de Narbona, debemos agradecer a nuestro amigo y colega Laurent Callegarin (Université de Pau et des Pays de l'Adour) las gestiones que nos permitieron acceder a la misma, gracias a la labor del conservador Ambroise Lasalle. Igualmente debemos mencionar a Asunción Gamecho su ayuda para contactar con el Museo de Béziers y a la conservadora Peggy Albert el envío de los datos del repertorio de ese Museo. La información de los hallazgos aislados fue proporcionada por P. P. Ripollés (Universidad de Valencia), Roberto

\section{NUEVO TESORO, NUEVOS HALLAZGOS}

El 27 de abril de 2006 durante la campaña de excavación en La Vega Baja de Toledo, tuvo lugar el hallazgo de un tesorillo visigodo formado por treintaiún tremises que van desde época de Sisebuto (612-621), representado con un solo ejemplar, pasando por Suintila (con 9), y Sisenando (12), hasta Chintila (636-639) (9), bajo cuyo reinado se cerraría el conjunto ${ }^{3}$. Se trata del único hallazgo que comprende este periodo ya que el tesoro de La Capilla se cerró en tiempos de Sisenando (631-636), mientras que el siguiente en cronología, el de La Grassa, alcanza el reinado de Recesvinto (653-672).

Este conjunto de Toledo contiene numerosas variantes de leyenda como sucede con el Sisebuto de $I s$ pali (cat. 276 e), también con algunas de las piezas de Sisenando, como en Acci (cat. 438 gg.1), las dos de Eliberri (cat. 458 m.1; 458 n.1), así como las de Tucci (cat. 426 k.1; 462 1.1) y Bracara (cat. 469 b.1). A nombre de Chintila y ceca de Barbi hasta la fecha solo se conocía un ejemplar por lo que este hallazgo proporciona otra variante de leyenda -que por ser más completa que la conocida pasaría a ser cat. 485 a.1-. Lo mismo sucede con respecto al taller de Eliberri, aunque en este caso se añaden dos variantes de leyenda a las dos ya conocidas (cat. 491 c. $1 ; 491$ d.1). Por su parte la pieza de Mentesa de este mismo monarca, añade una variante tipológica a las registradas (el tipo 3 e.1).

La pieza más destacada de Chintila, y probablemente del todo el conjunto, es sin embargo la acuñada en Elvora (cat. 496A.1) (fig. 1), taller desconocido hasta entonces para este monarca. De hecho sobre la base de los ejemplares conocidos, la ceca de Elvora parecería que cierra su producción en tiempos de Suintila (621-631), hasta una nueva apertura bajo el reinado de Wamba (672-680). Este ejemplar pone de manifiesto que su producción pudo continuar durante todos los reinado y que en un futuro podrían aparecer esos

Cortés (Universidad de Navarra), Álvaro Fernández Flores (Arqueología y Gestión, S.L., Sevilla) y Gabriel Carvajal (Sevilla). Por su parte José Antonio Herrero (S.A., Madrid) muy amablemente nos envío las fotografías y datos de dos interesantes piezas. De nuevo en este trabajo debemos mencionar a José Antonio Correa, Catedrático de la Universidad de Sevilla su disposición y apreciaciones a mis consultas y a J. C. Sánchez Pardo sus interesantes comentarios y sugerencias fundamentalmente sobre los talleres galaicos. Otras personas que queremos nombrar son Iago Urgorri (Revista Omni), Eduard Domingo (Áureo \& Calicó, Barcelona), y Peter Bartlett (Costa Rica), siempre dispuesto a ayudarnos en cualquier trabajo que emprendamos.

3. Sobre las vicisitudes del mismo, remitimos directamente al trabajo de los arqueólogos (Caballero, Maquedano \& Sánchez 2010). 

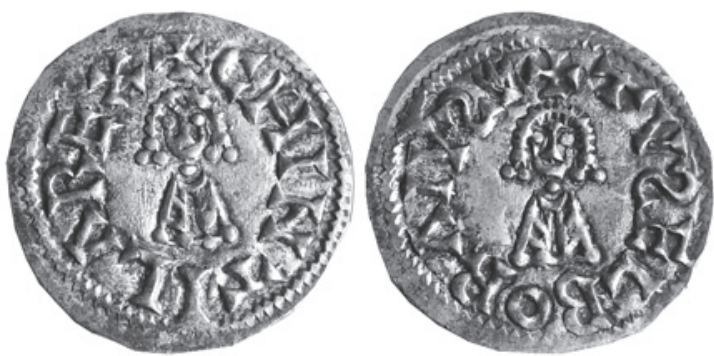

Figura 1. Tremis de Chintila de Elvora (Tesoro de La Vega Baja, Toledo).

especímenes, algo que sería extensible a cualquier taller visigodo 4 .

Por otro lado el ejemplar de Suintila acuñado en la ceca galaica de Cepis (cat. 412.2), vendría a confirmar otra moneda que responde a la misma descripción $-\mathrm{y}$ que incluso podría ser del mismo cuño- aunque en su momento, un exceso de celo nos llevo a considerarla algo sospechosa (Pliego 412.1) (fig. 2). (Ver tabla I).

A las pocas semanas de hallar el conjunto descrito, se encontró en la misma zona aunque de manera aislada un ejemplar de Leovigildo (569-586) y ceca de Toleto (cat. 42 c.14), al que habría que añadir otras piezas halladas en la misma Vega Baja de Toledo en otras campañas arqueológicas anteriores, así un Suintila de Tarracona (cat. 341 g.4), otra de Cordoba (367 a.19) de este mismo monarca, así como un Tulga también de Tarracona (cat. 509 b.1), y por último un Ervigio de $I s$ pali (cat. 649 f.7). Todas estas piezas han engrosado la colección numismática del Museo de Santa Cruz de

4. Este es un claro ejemplo del riesgo que se corre al extrapolar la metodología usada para otros periodos a la serie visigoda, y extraer conclusiones sobre temas como la circulación monetaria, o fundamentalmente, sobre volumen de producción basado en los especímenes conocidos, puesto que el corpus visigodo está continuamente experimentado transformaciones. En este sentido en Castro (2011), entre otros asuntos, se analiza "la evolución del volumen circulante", sin definir qué tipo de circulación habrían tenido los tremises en la Hispania goda. Por otro lado, nos sorprende las conclusiones a las que llega el autor, así como el que haya podido realizar la totalidad de sus figuras -sobre 'volumen de producción', 'volumen de emisión por provincias', 'evolución del peso del tremis', etc.-, basándose, tal como él mismo expone (Castro 2011: 226, nota 1), en los resultados obtenidos por los autores del CNV (2006) y por Marques et alii (1995), teniendo en cuenta que el primero de ellos es un muy completo catálogo de tipos en el que los pesos son expuestos como una horquilla entre el peso mayor y el menor de las piezas conocidas - que no son recogidas al completo-, y el segundo es un excelente ensayo, aunque en él se incluyen únicamente 238 ejemplares. Ninguna de esas obras incluye más datos para dichas estadísticas que la obra de Miles (1952).

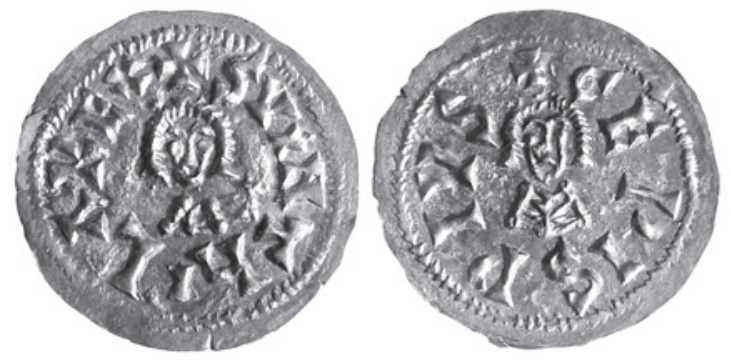

Figura 2. Tremis de Suintila de Cepis (Tesoro de La Vega Baja, Toledo).

Toledo, que hasta entonces fundamentalmente la conformaba el tesoro hallado en 1957 de tiempos de Egica \& Witiza (Pliego 2009, t. I: 249-50).

Aparte de los mencionados, procedente del mismo territorio perteneciente a la antigua Carthaginensis, hemos incluido también un ejemplar de Leovigildo y ceca de Elvora hallado en Recopolis (Castro 2008: 134, fig. 2), y en otro ámbito bien distinto, en territorio de la Gallaecia tuvo lugar el hallazgo de un tremis a nombre de Egica y ceca de Emerita (cat. 705.7) en la campaña arqueológica de 2008 en la ciudad de Chaves (Vila Real, Portugal) (Guedes 2006-2009).

Estos hallazgos son la prueba de un renovado impulso por parte de la Arqueología encaminado a situar el registro numismático en estratigrafía al nivel que debió tener siempre. No obstante, y a pesar de lo llamativo de estos descubrimientos, hoy por hoy aún continúan siendo muy escasos, por lo que se debe actuar con prudencia a la hora de extraer afirmaciones excesivamente categóricas en este sentido ${ }^{5}$.

Aparte de estos descubrimientos procedentes de excavaciones arqueológicas, nos ha llegado la noticia de varios hallazgos aislados y casuales que carecen de información estratigráfica, como es el caso de un ejemplar de Egica \& Witiza y ceca de Tarracona que presenta una leyenda no registrada hasta ahora (cat. 716 cc.1), o un Recesvinto de Toleto (cat. 580 c.12) hallado en Calahorra

5. Según Castro (2011: 239), los hallazgos de la Vega Baja "parecen confirmar la llegada, o concentración de tremises en la capital que ejerce por tanto, de núcleo acaparador de moneda, y tal vez, de centro último de transformación fiscal", conclusión que nos parece algo exagerada si tenemos en cuenta que estamos considerando un total de 35 piezas. El autor parece desconocer la existencia de los grandes hallazgos que ha proporcionado el sur de la Península, lo que le lleva a decir que "las razones que impulsaron el incremento de piezas en circulación en el primer tercio del siglo VII, no han sido todavía suficientemente resueltas", o que "el grueso de la producción se concentró en las cecas meridionales" (Castro 2011: 226, 230). 
Tabla I

\begin{tabular}{|c|c|c|c|c|}
\hline Reinado & Provincia & Ceca & $\mathrm{N}^{\mathrm{o}}$ ejempl. & cat. (Pliego) \\
\hline Sisebuto & Baetica & Ispali & 1 & 276 e. 1 \\
\hline \multirow{4}{*}{ Suintila } & Carthaginensis & Toleto & 5 & $\begin{array}{c}361 \text { a. } 51-53 ; 361 \text { b. } 14 \\
361 \text { d. } 17\end{array}$ \\
\hline & Baetica & Tucci & 2 & 387 n. $10 ; 387$ p.3 \\
\hline & Lusitania & Emerita & 1 & 393 b.61 \\
\hline & Gallaecia & Cepis & 1 & 412.2 \\
\hline \multirow{6}{*}{ Sisenando } & \multirow{3}{*}{ Carthaginensis } & $A c c i$ & 2 & 438 e. $2 ; 438$ gg. 1 \\
\hline & & Castilona & 2 & 439 c. $2 ; 441$ f. 2 \\
\hline & & Toleto & 3 & 449 a. $2 ; 449$ b. $5 ; 449$ f.7 \\
\hline & \multirow{2}{*}{ Baetica } & Eliberri & 2 & $458 \mathrm{~m} .1 ; 458 \mathrm{n} .1$ \\
\hline & & Tucci & 2 & 426 k.1; 4621.1 \\
\hline & Gallaecia & Bracara & 1 & 469 b. 1 \\
\hline \multirow{5}{*}{ Chintila } & Carthaginensis & Mentesa & 1 & 481A.1 \\
\hline & \multirow{2}{*}{ Baetica } & Barbi & 1 & 485 a. 1 \\
\hline & & Eliberri & 2 & 491 c. $1 ; 491$ d. 1 \\
\hline & \multirow{2}{*}{ Lusitania } & Elvora & 1 & 496A.1 \\
\hline & & Emerita & 3 & 499 a. $5 ; 499$ d. $5-6$ \\
\hline \multicolumn{3}{|c|}{ Total } & 30 & \\
\hline
\end{tabular}

mientras se realizaban labores agrícolas, o bien un fragmento aparecido en la zona de Salteras (Sevilla) que tras un numerosas vacilaciones hemos llegado a la conclusión de que se trata de una moneda de Chindasvinto acuñada en el taller de Eliberri (cat. 542 b.1). Esta pieza además nos da la oportunidad de comentar otro ejemplar del mismo reinado y ceca, que sería el segundo de los citados bajo nuestra entrada 542 -la primera pertenece a la colección del British Museum-. Nos referimos a una moneda conservada en el Museo de Martos y cuyo anverso fue publicado por Recio (1999: 750, 3) aunque no pudimos acceder a ella ni físicamente ni a través de una fotografía de calidad. Cuando se ha tenido esta oportunidad, hemos podido comprobar que se trata de una falsificación, a pesar de que en el mencionado artículo se dice que fue hallada en Estepa (cat. 1136A).

\section{NUEVO TALLER, NUEVA UBICACIÓN}

Aunque la nómina de los reyes visigodos parece que quedó fijada tras la incorporación de Recaredo II -de quien hasta los años 90 no se conocían ejemplares-, al que se debe añadir los, con toda probabilidad usurpadores,
Iudila y Sunifredo -ambos conocidos gracias a la existencia de monedas a sus respectivos nombre-, el elenco de las ciudades que acuñaron a nombre de algún rey visigodo ha crecido de un modo espectacular en los últimos años, pasando de 79 conocidas en tiempos de Miles (1952) a 96 a día de hoy, incluyendo la que aquí se menciona. Huelga decir que las que más interés han suscitado son aquellas que únicamente se encuentran registradas en documentos antiguos -como sería el caso de aquellas mencionadas en el Parroquial suevo (David 1942)- o el más reciente de la Tábula del Bierzo (ver Villanueva \& Cavada 2001)-, y que en su práctica totalidad aluden a lugares pertenecientes a la provincia visigoda de Gallaecia.

El caso del ejemplar que queremos comentar es una moneda a nombre de Suintila y la leyenda alusiva al taller monetario presenta la forma de VADA. La pieza muestra la tipología que representa el busto del monarca vestido con la cota de malla o hamata muy simplificada, en combinación con la lórica torácica que aunque originaria del taller de Emerita fue elegida habitualmente por algunas cecas galaicas, combinándose principalmente con otros tipos más sencillos (fig. 3).

Ya se ha señalado en otro lugar (Pliego 2011: 87 ss) que esta combinación tipológica fue utilizada en un área 
de dispersión importante que no ayuda a la hora de localizar el taller de $V A D A$ en una población actual. No obstante en ese trabajo planteamos varias posibilidades. En principio parece poco probable su identificación con la actual Vada ubicada en Santander, ni con la Bada de San Juan de Parres, Asturias, puesto que a pesar de su completa coincidencia toponímica y de la relativa cercanía de estos lugares con las cecas de Saldania y Mave, la utilización de la tipología torácica resulta extraña en este ámbito en el que los talleres mencionados presentan una tipología bastante homogénea. Aunque no es prudente descartarlas totalmente, ya que se han constatado importantes excepciones al argumento tipológico ${ }^{6}$, es más probable su localización en alguna de las localidades portuguesas que incluyen Vade en su topónimo, ya sea en Sao Tomé o Sao Pedro de Vade, ambas fregresías de Ponte da Barca, en el Distrito de Viana do Castelo 7 . Ambas localizaciones se encontrarían en el entorno de $\mathrm{Bra}$ cara por lo que se trataría de identificaciones fácilmente asumibles desde una perspectiva histórica.

A pesar de lo dicho, existe un interesante topónimo proporcionado por Martín Sarmiento en su $\mathrm{Ca}$ tálogo de voces y frases de la lengua gallega escrito entre 1745 y $1755^{8}$. Bajo el epígrafe 'Voces gallegas antiguas', en concreto en un documento de 1220 se recoge: "In ipso molino qui stat in ribulo Tomeza citra Mollia Vada prope villam de Ponteveteri". El término al que nos referimos es el de Mollia Vada, del que el autor dice que es Mollavau, en la actualidad Mollabao, que tal como dice el texto, es un barrio en la propia provincia de Pontevedra. Vistas las distintas opciones posibles, y sin negar que pudieran existir otras, quedaría la cuestión abierta a ulteriores investigaciones que nos lleve a una certera localización.

Queremos detenernos también en la ceca de Laure puesto que si bien no tenemos piezas nuevas sobre la misma, si se han obtenido datos más recientes sobre

6. Como sería el caso de unas emisiones de Sisebuto en la ceca bética de Eliberri en las que, casualmente y como única excepción, se utiliza la lórica torácica en sus reversos (Pliego nº 273).

7. De las localizaciones propuestas en el catálogo de subastas donde fue publicada la pieza (Künker, 26 de septiembre de 2011, lote $\mathrm{n}^{\circ} 1101$ ), se ha obviado Portela de Vade en el distrito de Braga, ya que según Machado (ver bibliografía) en el año 1058 ese lugar se denomina Vanade, topónimo sin relación de continuidad con Vada. (ver Pliego 2011: 88, nota 21).

8. Este religioso erudito además de destacar por sus conocimientos de Botánica estaba interesado por la lengua gallega en su más amplia concepto. Para ello realizó varios viajes a Galicia en los que según Pensado (1973: 40) Sarmiento no solo recogía los lugares por los que pasaba sino los que se encontraban a derecha e izquierda registrando el nombre de montes, ríos valles, etc.

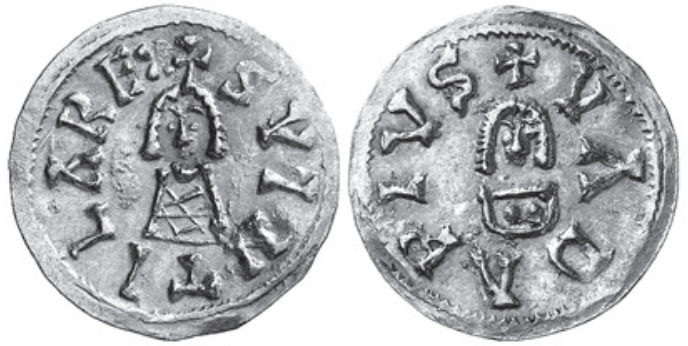

Figura 3. Tremis de Suintila de Vada (Künker Auktion, 2011).

su posible ubicación. Es una de las cecas cuyo intento de esclarecimiento ha resultado más complejo ya que, como explicamos en otro lugar (Pliego 2009: 134135), se ha considerado que podían entrar en juego varias formas toponímicas que tradicionalmente se han relacionado con Laure, así Lauruclo (Beltrán 1915: 129-130) y Lavrncio (Bouza Brey 1942: 361) que podría a su vez estar identificado con Lavrencio//Labrentio (Pliego 2009: 134). No obstante compartimos la opinión de J. A. Correa quien considera que Laure, que solo aparece de este modo en un ejemplar de Sisebuto (Pliego 311 a.1), debe ser una localidad diferente ya que resulta complejo admitir la simplificación del topónimo por suspensión. Recientemente J. C. Sánchez Pardo nos ha sugerido que existe un documento del monasterio de Samos datado hacia el año 853 en el que se habla del territorio de Laure (Lucas 1986: 135$\left.137, \mathrm{n}^{\mathrm{o}} 142\right)^{9}$. Este territorio se encontraría en el valle del río Lor, es decir, la zona de O Caurel, cerca de $\mathrm{Ca}$ rioca (Quiroga) y de Incio, esta última también ceca visigoda en tiempos de Sisebuto con letrero Inceio.

\section{LAS ADICIONES DE COLECCIONES DE MUSEOS E INSTITUCIONES}

Son varias las colecciones que suponen una novedad en el corpus visigodo. La más numerosa es la del Museo Romano de Mérida que está compuesta por 81 ejemplares. Aunque en comparación con la mayoría de las colecciones españolas es una cifra importante, su conformación, sin embargo, es bastante reciente y las piezas no proceden, como suele ser habitual en estos casos, de

9. “...sancti Iohanis cum omnia quicquid ibidem domnus Adefonsus concessit; in Laure monasterium Suveretum; ecclesiam in Kairola et villam; in Iurres, terra...". En el siglo X debía seguir conservando ese nombre según puede verse en el mapa de los territorios altomedievales en los siglos IX-XI ilustrado por Baliñas (2000). 
hallazgos de la zona sino que han sido adquiridos en subastas numismáticas. A excepción de un Recaredo I de Ispali -que resulta ser una variante de leyenda no registrada (cat. 106 g.1)-, todas las monedas son de la ceca de Emerita, contándose con piezas de Recaredo I (4 ejemplares), Liuva II (3), Witerico (3), Gundemaro (2), Sisebuto (28), Recaredo II (1), Suintila (26), Sisenando (1), Chintila (1), Wamba (1), Ervigio (1), Egica (1) y del reinado conjunto de Egica \& Witiza (6) (fig. 4). Como es posible apreciar, el peso del repertorio recae en los reinados de Sisebuto y Suintila, coincidiendo con lo que sucede con el corpus general. De hecho la pieza más común del mismo es precisamente Suintila de Emerita. Pocas novedades sin embargo encontramos en la colección, si exceptuamos el mencionado Recaredo I de Ispali al que podríamos añadir un ejemplar de Recaredo I (cat. 113 e.1), y otro de Suintila (cat. 393 bb.1), ambos, obviamente, del taller de Emerita.

Una única pieza visigoda posee el Museo de Jaén que ahora presentamos aquí. Se trata de una moneda acuñada a nombre de Wamba y ceca de Toleto que, según los datos proporcionados por el personal del $\mathrm{Mu}$ seo fue hallado en la Plaza de Cambil, $n^{\circ} 2$ en la misma ciudad de Jaén (cat. 618 e.38).

Uno de las colecciones más interesantes de los que se incluyen es quizás la del Musée de Narbonne debido a la complejidad que supone casar la bibliografía antigua con el repertorio actual del Museo que es similar, como veremos, a lo que sucede con el Musée du Biterrois en Béziers. El Musée de Narbonne aparece mencionado en su casi totalidad en nuestro corpus, si bien en su momento no pudimos tener acceso directo a las piezas, ni siquiera a través de fotografías, por lo que en él nos limitamos a utilizar los datos de Miles (1952) ${ }^{10}$. Afortunadamente, gracias a las gestiones realizadas por nuestro amigo y colega Laurent Callegarin, así como a la cortesía del conservador de dicho Museo, Ambroise Lassalle, hoy tenemos la oportunidad de detallar las monedas del Museo de Narbona subsanando algunas confusiones del pasado.

La bibliografía alusiva a la colección numismática visigoda de Narbonne - principalmente la obra de Belfort (1892)- llevó a Miles (1952) a incluir diez ejemplares en su corpus a cuya relación, por nuestra parte, añadimos un hallazgo acaecido en esa ciudad, en concreto de Tulga y ceca de Tirasona, dato que debió escapársele a Miles puesto que fue publicado por Amardel (1896). No obstante son solo ocho las piezas que componen en la actualidad la colección de Narbona, tal

10. Tampoco conocíamos entonces la publicación de J.-Cl. Richard (2005) en una revista de ámbito local que nos hubiera sido de gran ayuda.

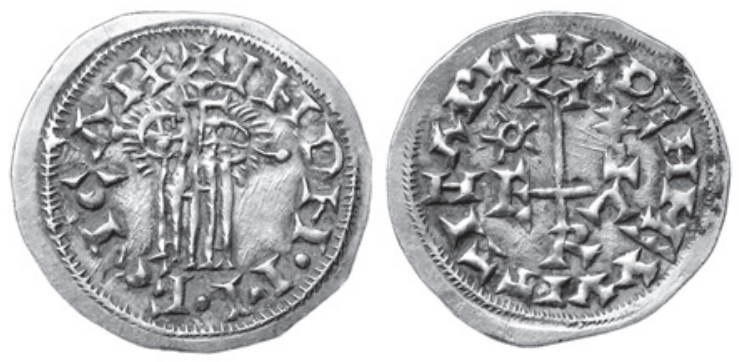

Figura 4. Tremis de Egica \& Witiza (Museo de Arte Romano de Mérida).

como se nos ha informado recientemente, que es el repertorio que recoge Richard en 2005. Resumiendo el asunto, da la impresión de que la multiplicación de trabajos alusivos a las mismas piezas, sobre todo en lo que respecta a G. Amardel, en momentos diferentes y sin contar con el importante testimonio que supone una fotografía del ejemplar -aunque un buen grabado o el número de inventario del Museo también hubiera servido-, ha confundido a los autores posteriores.

Las piezas que aparecen mencionadas por todos los autores y que no presentan problemas son cuatro: Recaredo I de Narbona (cat. 64 b.2); Recaredo I de Elvora (cat. 112 a.12); Chindasvinto de Narbona (cat. 526 a.2) y Egica de Narbona (cat. 662 c.3). Aparte hay un Ervigio de Narbona (cat. 631 b.7), que Miles duplica en dos entradas diferentes (Miles 396 a.7 y Miles 396 c.2) ${ }^{11}$, error repetido por quien escribe (Pliego 631 b.7 y Pliego 631 c.2). Ya se ha mencionado que existe un ejemplar de Tulga y ceca de Tirasona hallado en Narbona que fue publicado por Amardel en 1896, pero que Miles no recogió (cat. 510 a.1). Por otro lado en Richard (2005, $\mathrm{n}^{\circ}$ 50) entra en escena una pieza de Leovigildo de Toleto no mencionada hasta entonces (cat. 43 c.36).

En lo que respecta a Agila II parece haber unanimidad en la existencia de una moneda de Agila II y a pesar de la diferente descripción e incluso de la disparidad de pesos, las dos entradas de Miles (513 a y 513 c, que se corresponden con Pliego 795 b y 795 c) son fácilmente asimilables entre sí y ésta es la pieza que actualmente se encuentra en el Museo de Narbona (fig. 5). No obstante en 1916 Amardel presentó "Un triens inédit d'Achila",

11. Debido no solo a que utiliza referencias diferentes alusivas a la misma pieza -y por tanto diferente descripción de leyenda y de dibujo-, sino que además en su entrada (Miles 396 c.2), intercambia entre sí los pesos de las dos monedas citadas, que recoge de Belfort $(1892,3162)$ - una del Museo de Narbona $(1,40)$ y otra de la col. Pont d'Amécourt $(1,15)$ - por lo que, no identifica la de Narbona con la pieza ya citada en su otra entrada (Miles 396 a.7) que pesa 1,40 g. 


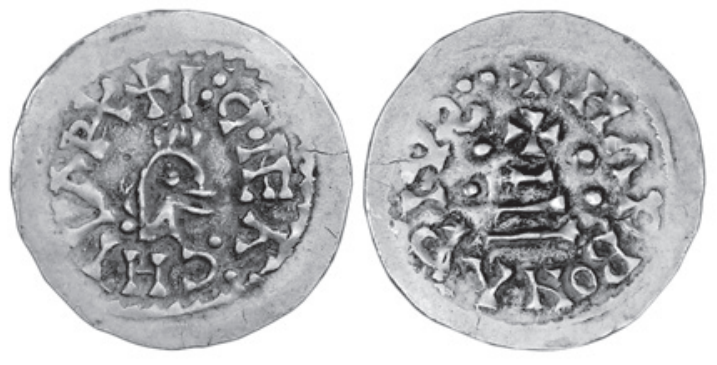

Figura 5. Tremis de Agila II de Narbona (Musée de Narbonne).

que había sido hallado en Castel-Rousillons (cat. 796 a.1). Este ejemplar, tal como puede verse en la ilustración de dicho artículo, presenta una tipología diferente al anterior - con busto de frente- $y$ un peso de 1,30 frente a los 1,46 g del otro. Richard (2005, $\left.\mathrm{n}^{\circ} 56\right)$ confunde las referencias de ambas piezas, ya que mientras que el único ejemplar de Narbona de ese Museo presenta busto de perfil y pesa $1,46 \mathrm{~g}$, cita sin embargo los datos de la otra, en lo que respecta a lugar de hallazgo, Ruscino, y a su bibliografía. Este tremis no se encuentra en la actualidad en el Museo de Narbona aunque Amardel dice que "a été acquise par M. Paul Thiers, mon collègue à la Commission archéologique de Narbonne, et généreusemnet offerte par lui au Musée".

También de Belfort (1892) proceden las referencias de las monedas visigodas del Musée du Biterrois, Béziers -o a la Sociedad Arqueológica de esa misma ciudad-recogidas por Miles: un ejemplar de Chindasvinto de Narbona (Belfort 1892, $\mathrm{n}^{\circ} 3150$ ) y tres de Agila II de Narbona (Belfort 1892, no 3155, 3158 y 3159). Mientras que la alusión de Chindasvinto no presenta ningún problema y ha sido constatada en dicho museo recientemente (cat. 526 aa.1), las referencias a Agila II encierra cierta confusión. Parece claro que los números 3155 y 3159 aluden al mismo ejemplar (cat. 795 d.1), como así muestra la coincidencia de los pesos, habiendo producido el equívoco las distintas procedencia de las referencias bibliográficas de Belfort cuyos grabados -0 dibujos- no debían ser exactamente iguales, sobre todo en lo que respecta a las leyendas. Por su parte la tercera de las referencias de Belfort $\left(1892, \mathrm{n}^{\circ} 3158=\right.$ Miles 513 $\mathrm{e}=$ Pliego 795 e) presenta unas leyendas extrañas, mostrando en anverso media leyenda del reverso y viceversa, lo que hacía pensar en un error del cuño. No obstante aunque en el Musée du Biterrois de Béziers solo hay una moneda de Agila II, la fotografía enviada por la conservadora de ese Museo explica esa confusión de Belfort. La pieza en cuestión se encuentra fragmentada en tres

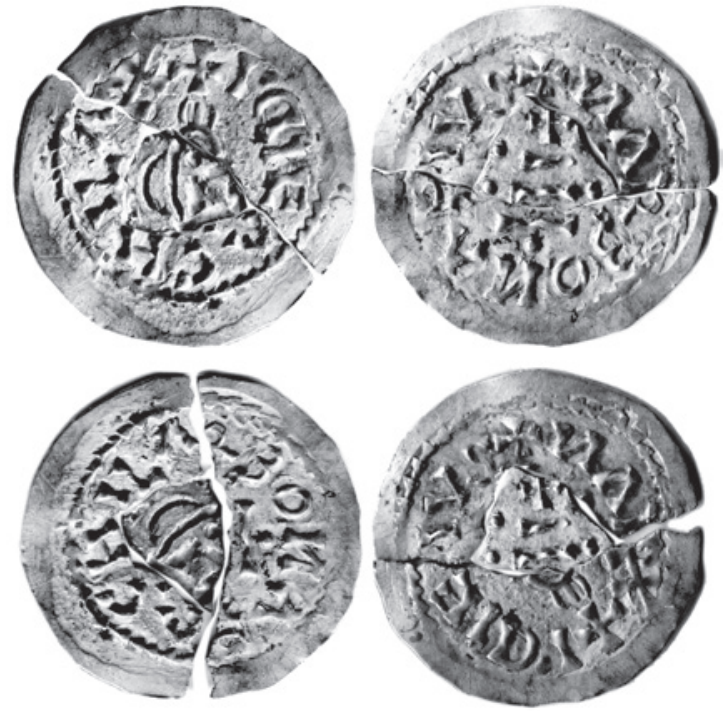

Figura 6. Tremis de Agila II de Narbona en posición correcta (arriba) y errónea (abajo)(Musée du Biterrois, Béziers).

trozos y su inexacta disposición, en la que se mezclan partes del anverso y del reverso, debe venir de antiguo lo que ha llevado a que el error se haya perpetuado (fig. 6).

\section{OTRAS NOVEDADES MONETARIAS VISIGODAS DESTACADAS}

Aparte de las comentadas en cada uno de los apartados anteriores, presentamos otras novedades que como se adelantó proceden en su mayoría de ejemplares procedentes de subastas numismáticas. Una de las monedas más interesantes de las que incluimos en este trabajo pertenece al reinado de Hermenegildo, y ha sido subastada recientemente en una importante Casa de subastas portuguesa. La pieza muestra un buen estado de conservación y una fábrica impecable. Aunque responde a una descripción recogida en nuestro corpus (Pliego 62 b), del único ejemplar conocido bajo esa entrada, perteneciente a la antigua colección de Vidal Quadras -hoy en paradero desconocido- solo se conserva un grabado en la obra de Heiss (1872, Pl. II, 1). Como es posible observar en la fig. 7 ambas monedas son idénticas aunque la forma del cospel sobrante, diferente en cada una de ellas, revelaría que no se trata de la misma pieza. Con ella se eleva el número de monedas conocidas a nombre de Hermenegildo a ocho, viniendo a engrosar la tipología con leyenda INCLITVS REX en cinco, ya que del tipo, más escaso, con leyenda REGIA DEO VITA solo se cuenta con la referencia de tres piezas. 


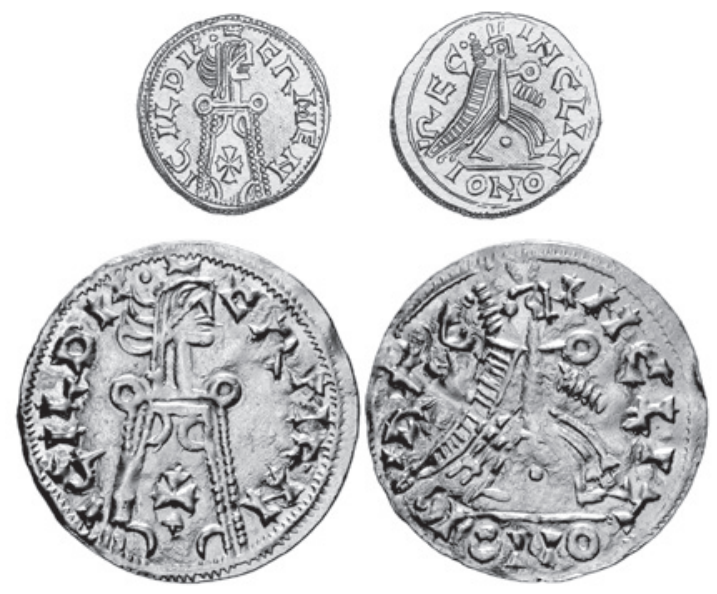

Figura 7. Tremis de Hermenegildo (Numisma Leilões, 2011).

Aunque muchos de los ejemplares incluidos aquí presentan novedades tipológicas y epigráficas, entre ellos podemos destacar una moneda de Sisebuto de Gerunda (cat. 250A), que en lugar del tipo tarraconense más habitual con paludamentum, presenta la tipología propia de Barcinona. Por otro lado gracias a la inclusión del Musée de Narbonne conocemos el ejemplar de Tulga de Tirasona, si bien casualmente hace muy poco nos llegaron los datos de otro ejemplar del mismo monarca y ceca aunque con una leyenda algo diferente (cat. 510 b). Con respecto al ejemplar de Chindasvinto de Eliberri (cat. 542 b), es una lástima que solo se haya conservado un fragmento ya que únicamente se conoce una moneda similar en el British Museum. En lo que respecta a la tipología, no conocíamos hasta la fecha una pieza de Wamba de Cordoba con el busto de perfil clásico (tipo 1 c) (cat. 618A), ni de Witiza de Toleto que presentara el tipo de perfil con cetro (cat. 771A).

Otras precisiones que nos permiten completar nuestro corpus viene de manos de otros especímenes. En nuestro trabajo de 2009 incluimos una moneda de Chintila de Castilona que aunque fragmentada casi a la mitad presentaba una tipología diferente a las conocidas (Pliego 478.1) y que ahora hemos podido confirmar gracias a un nuevo ejemplar completo (cat. 478.2). De este mismo monarca y ceca de Barbi solo se conocía una moneda por lo que la presente en el tesoro de La Vega Baja de Toledo, que responde además a otra descripción, hace que contemos ahora con dos variantes (cat. 485 a y 485 b). Algo similar sucede con un Egica de Eliberri que mostraba el tipo (16 c) y al que se añade ahora otra variante. En ambos casos se incluye la referencia de la pieza conocida anteriormente que, en los dos casos, pasa a ser la variante (b).

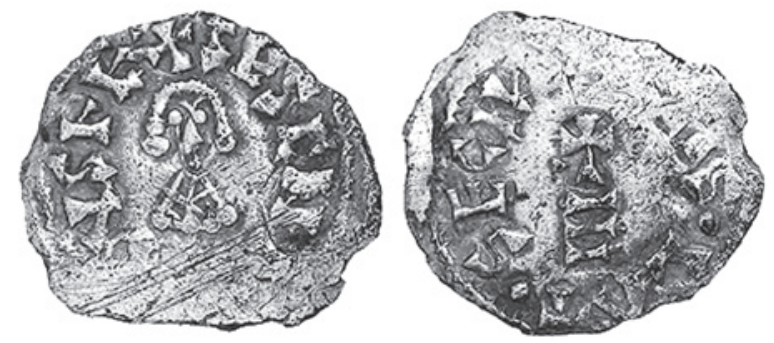

Figura 8. Tremis de Sisenando (falsificación de la época) (Archivo Herrero S. A.).

Se incluyen igualmente numerosas variantes de leyendas para las que remitimos directamente al Anexo. No obstante quisiéramos mencionar un interesante ejemplar que parece ser una falsificación de época (cat. 814A). La leyenda de anverso alude al rey Sisenando - en la habitual forma de Sesenandus-, y tanto ésta como el tipo son propios de esa época (631-636), siendo muy similar a una de las tipologías que presenta ese monarca en el taller de Toleto (Pliego 449). No obstante la tipología de reverso es la 'cruz sobre gradas', abandonada hacia 584, en tiempos de Leovigildo, y que aunque fue recuperada posteriormente, esto no sucedió hasta el reinado conjunto de Chindasvinto \& Recesvinto (649-653). A esa rareza se le añade el letrero que puede leerse que alude a una $L A T$. SECIN, que recuerda las piezas suevas con leyenda Latina MVNita. Por nuestra parte no hemos hallado de momento ninguna población que podamos identificar con dicho letrero si bien seguimos investigando y confiamos que un futuro próximo arroje algo de luz a este asunto.

\section{CORRIGENDA}

Aparte del Chindasvinto de Eliberri conservado en el Museo de Martos (Jaén) que se ha comentado, quisiéramos detenernos en otra pieza. Se trata de un ejemplar de Roderico de Egitania perteneciente a la colección de la Hispanic Society of America -y procedente de la antigua colección Ferreira- que no se encontraba en la American Numismatic Society cuando estudiamos aquel repertorio. Un exceso de celo nos llevó a revelar una pequeña duda con respecto a esta moneda en forma de pregunta a pie de página - “Se trata de una falsificación?"-. No obstante más adelante tuvimos acceso a una fotografía de calidad que eliminó esa duda, algo que ya mencionamos en otro lugar (Pliego 2010: 97). 


\section{ANEXO I}

a La moneda visigoda (Sevilla 2009)

\section{LEOVIGILDO}

Toleto

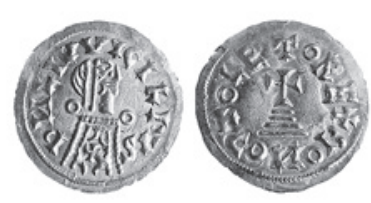

$42 \mathrm{c} .14$

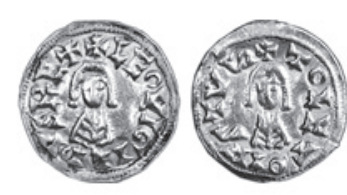

$43 c .36$

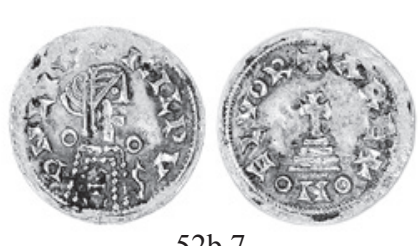

52b. 7

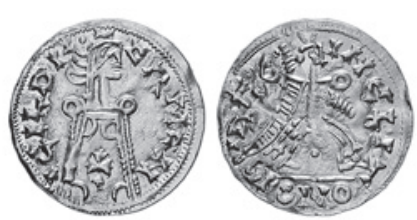

$62 \mathrm{~b} .2$

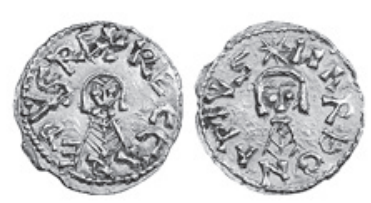

$64 b .2$

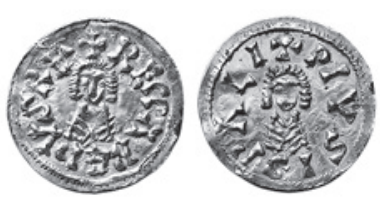

106g.1 


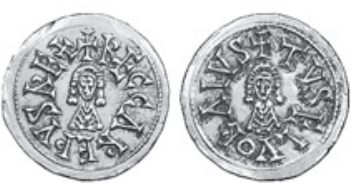

112a.12

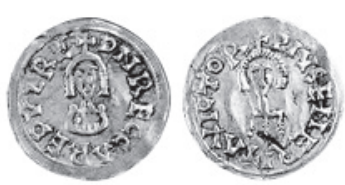

113 e. 1

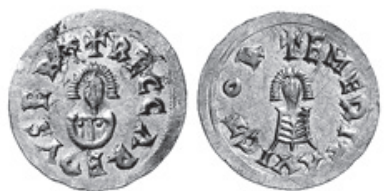

114b. 17

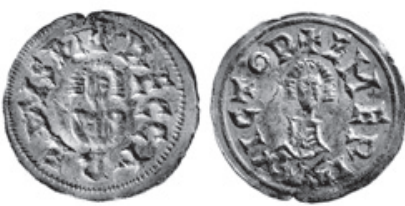

114g.29

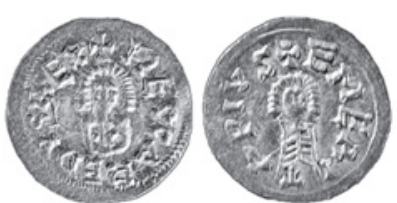

$116 \mathrm{a} .3$

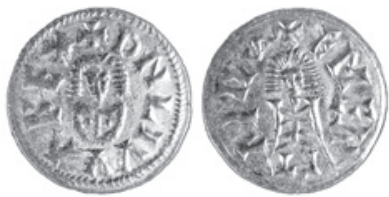

165a. 14

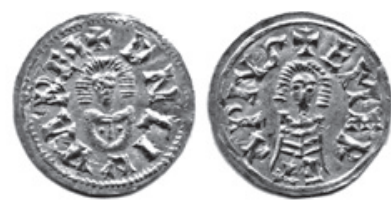

$165 \mathrm{c} .4$

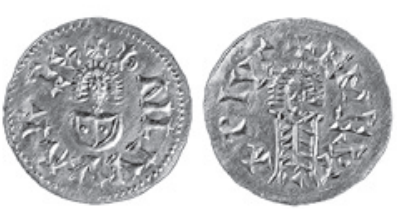

165 e. 3

Elvora

112

(a)

a) +RECC $\Lambda$ REDVSRE+

+ TVSELVOR $\Lambda$ IVS

12* $(1,44)$ Miles 90 a $(11 / 15)=$ Amardel 1890, $6=$

$=$ Elias 1950, $100=$ Richard 2005, 52* (M. Narbonne).

113 (e) +RECC $\Lambda$ REDV2RE

+ PIVSEMERITAVICTOR

1* (1,47) Áureo (16-12-1999) 2112* (M. Mérida, 37142).

114 (b) +RECC $\Lambda$ REDVSRE ${ }^{\mathrm{X}}$

+EMERIT $\Lambda$ VICTOR

16 (1,80) Vico (13-11-2008) 428* (M. Mérida 37746).

17* $(1,49)$ Áureo (1-7-2011) 177* (M. Mérida $\left.{ }^{12}\right)$.

(g) +RECC $\Lambda$ REDVSRE

+ EMERIT $\Lambda$ VICTOR

29* $(1,46)$ Vico (9-6-2005) 439* =

$=[\mathrm{CNV}$ 105.7 $]$ (M. Mérida, 37555).

116 (a) +RECC $\Lambda$ REDVSREX

+EMERI $|\mathrm{T}| \Lambda \mathrm{PIVS}$

$3^{*} \quad(1,48)$ Hervera (18-11-2008) 1389* (M. Mérida, 37737).

\section{LIUVA II}

Emerita

165
(a) +DNLIVV $\Lambda R E X$
+EMER $|\mathrm{ET}| \Lambda \mathrm{PIV} \backsim$
14* (1,47) Vico (16-11-2006) 522* (M. Mérida, 37610).

(c) +DNLIVV $\Lambda R E+$

+EMER $\mid$ ET $\mid \Lambda$ PIV $s$

4* (1,50) Hervera (21-3-2000) 263* (M. Mérida, 37179).

(e) + DNLIVV $\Lambda$ RE

+ EMERI $|\mathrm{T}| \Lambda \mathrm{PIVS}$

3* (1,48) Vico (2-4-2009) 1357* (M. Mérida, 37784).

\section{WITERICO}

Emerita

194 (c) +VVITIRICVSRE+

+ EMERE $|\mathrm{T}| \Lambda \mathrm{PIVS}$

7 (1,50) Vico (9-6-2005) 440* (M. Mérida, 37556).

13* (1,44) Áureo (21-5-1998) 458* (M. Mérida, 37081).

14 (1,47) Áureo (22-9-2003) 368* (M. Mérida, 37428).

12. Aún no se le ha asignado número de identificación. 


\section{GUNDEMARO}

\section{Emerita}

231 (a) + GVNDEM $\Lambda R^{2}$ SRE $^{\mathrm{X}}$

+ EMERET $\Lambda$ PIVS

9 (1,43) Áureo (21-5-1998) 460*(M. Mérida, 37078).

10* (1,52) Áureo (16-12-2002) 439* (M. Mérida, 37417).

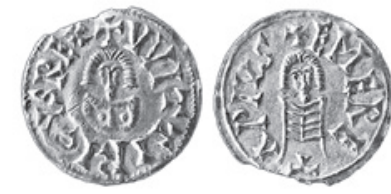

$194 c .13$

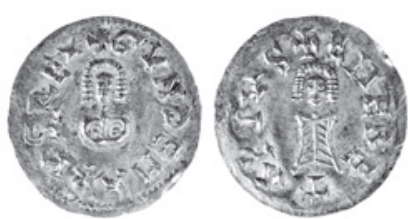

231a.10

$\begin{aligned} 250 \mathrm{~A} & + \text { SISEBVTVSREX } \\ & * \text { JERVN } \Delta \text { AIVSTV } \\ & 1 * \quad \text { Vico (3-3-2011) } 436^{*} .\end{aligned}$

Cordoba

\section{(k) t $s$ I $\backsim$ IBOTV $\backsim R E+$ \\ $+\mathrm{COR} \triangle \mathrm{DOB} \Lambda \mathrm{PIV} 2$ \\ 1* $\quad(1,47)$ Áureo (30-11-2011) 1197*.}

Ispali (e) $+{ }^{\bullet} \mathrm{SISEBVT}{ }^{\bullet} \mathrm{V}^{\bullet} \mathrm{SRE}{ }^{\bullet}$

$+\left[^{\bullet}\right] \mathrm{ISP} \Lambda$ LIPIVS $\bullet$

1* $\quad(1,42)$ Caballero, Maquedano \& Sánchez 2010, 2* (T. Vega Baja; M. Sta. Cruz Toledo, 2005/53/992).

Emerita

284 (a) + SISEBVTVSREX $^{\mathrm{X}}$

+EMERIT $\Lambda$ PIVS

10 (1,51) Hervera (10-7-2008) 498* (M. Mérida, 37692).

11 (1,50) Hervera (31-3-2009) 424* (M. Mérida 37767).

$12 * \quad(1,49)$ Áureo (1-7-2011) 182* (M. Mérida $\left.{ }^{13}\right)$.

(b) + SISEBVTVSRE ${ }^{\mathrm{X}}$

+EMERIT $\Lambda$ PIVS*

$12 * \quad(1,48)$ Áureo (4-7-2000) 143* (M. Mérida, 37200).

285 (a) + SISEBVTVSRE $^{\mathrm{X}}$

+ EMERI $|\mathrm{T}| \Lambda \mathrm{PIVS}$

34 (1,52) Áureo (16-12-1999) 2117* (M. Mérida, 37061).

35 (1,47) Durán (29-4-1998) 742* (M. Mérida, 37065)

36 (1,52) Durán (17-12-1997) 1352* (M. Mérida, 37141).

37 (1,44) Hervera (28-11-2000) 269* (M. Mérida, 37243)

38 (1,44) Hervera (10-12-2002) 889* (M. Mérida, 37395).

13. Aún no se le ha asignado número de identificación.

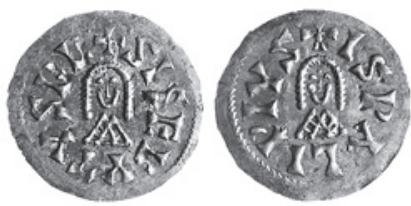

$276 \mathrm{e} .1$

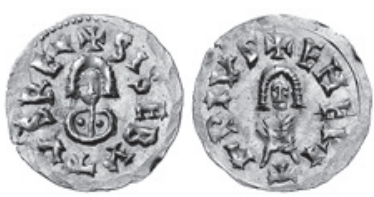

284a. 12

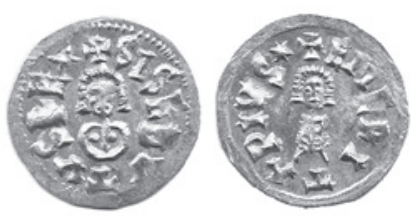

284b. 12 


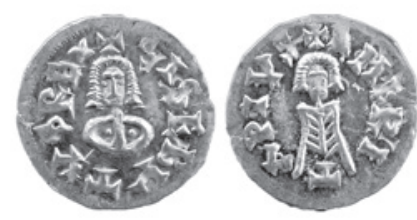

$285 \mathrm{a} .41$

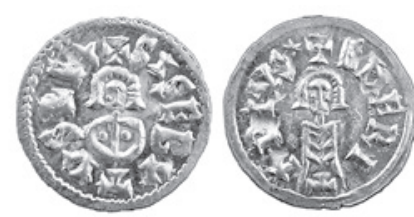

285 c. 61

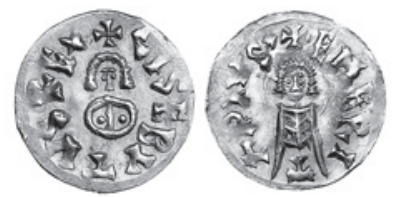

$285 \mathrm{cc} .1$

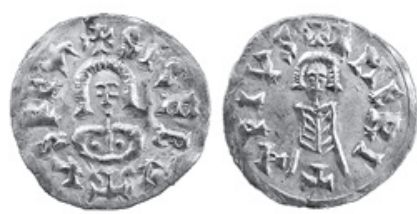

285 dd. 1

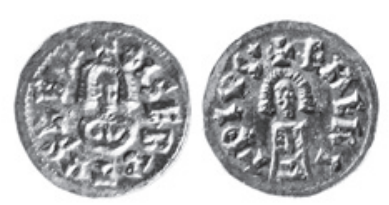

285g. 1

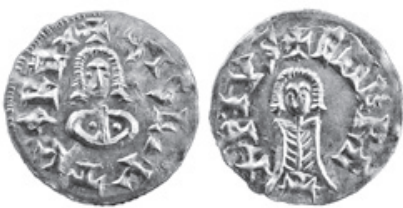

286b.23

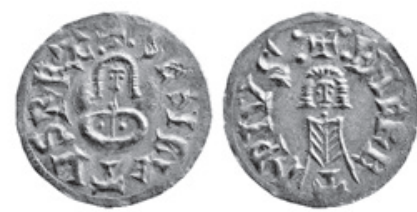

286g.6

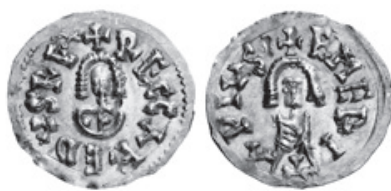

$335 \mathrm{a} .8$

39 (1,39) Áureo (22-9-2003) 371* (M. Mérida, 37429).

40 (1,39) Vico (10-6-2004) 288* (M. Mérida, 37475).

$41 * \quad(1,47)$ Hervera (10-7-2008) 500* (M. Mérida, 37694).

42 (1,43) Hervera (18-11-2008) (M. Mérida, 37738).

(c) + SISEBVTVSRE ${ }^{\mathrm{X}}$

+EMERI $|\mathrm{T}| \Lambda$ PIVS*

52 (1,49) Áureo (21-5-1998) 466* (M. Mérida, 37077).

53 (1,47) Áureo (21-5-1998) 467* (M. Mérida, 37079).

54 (1,48) Áureo (1-3-2000) 1178* (M. Mérida, 37167).

55 (1,53) Áureo (22-9-1999) 317* (M. Mérida, 37125).

56 (1,47) Áureo (26-1-2000) 330* (M. Mérida, 37165).

57 (1,53) Áureo (4-7-2000) 142* (M. Mérida, 37199).

58 (1,46) Hervera (10-12-2002) 890* (M. Mérida, 37393).

59 (1,48) Hervera (10-12-2002) 891* (M. Mérida, 37394).

60 (1,46) Herrero (16-10-2003) 402* (M. Mérida, 37432).

$61 * \quad(1,44)$ Hervera $(10-7-2008) 501 *$ (M. Mérida, 37695).

62 (1,44) Vico (2-4-2009) 1358* (M. Mérida, 37785).

(cc) t $\backsim$ ISEBVTVSRE ${ }^{\mathrm{X}}$

+EMERI $|\mathrm{T}| \Lambda$ PIVS*

1* Vico (1-3-2012) 373*.

(dd) + SISEBVTVSRE 8

+ EMERI $|\mathrm{T}| \Lambda$ PIVS

1* $\quad(1,48)$ Hervera (10-7-2008) 499* (M. Mérida, 37693).

(g) + SISEBVTVSREX •

+EMERI $|\mathrm{T}| \Lambda$ PIVS*

$1^{*} \quad(1,49)$ Hervera $(9-7-2002)$ (M. Mérida 37739).

(b) + SISEBVTVSRE ${ }^{\mathrm{X}}$

+EMERE $|\mathrm{T}| \Lambda$ PIVS

18 (1,50) Vico (9-6-2005) 448* (M. Mérida, 37557).

$23 *$ (1,57) Hervera (10-7-2008) 502* (M. Mérida, 37696).

(g) + :SESIBVTVSREX :

+ :EMERE $|\mathrm{T}| \Lambda \mathrm{PIVS}$ :

6* (1,46) Vico (9-6-2005) 449* (M. Mérida, 37558).

\section{RECAREDO II}

Emerita

335 (a) +REC $\Lambda$ REDVSRE ${ }^{\mathrm{X}}$

+ EMERI $|\mathrm{T}| \Lambda$ PIVS :

$8^{*} \quad(1,44)$ Áureo (28-5-2008) 105* (M. Mérida 37705). 
Tarracona

341

Acci

\section{SUINTILA}

(g) + SVIKTHIL $\Lambda$ RE

$+\mathrm{COPIV} \cdot \mathrm{T} \Lambda \mathrm{RR}$.

4* $\quad(1,4)$ García, Gómez \& Rojas 2007: 123, 131* =

= Caballero, Maquedano \& Sánchez 2010, 32* (H: La Vega

Baja; M. Sta. Cruz Toledo 20.007).

347 (bb) + SVINTHIL $\cdot$ RE

$+\operatorname{IVSTVSH}: \Lambda: \mathrm{C}$.

1* Vico (3-3-2011) 440*.

348 (dd) + SVINTII. $\Lambda \mathrm{R}^{14}$

$+\operatorname{IV} 2 T V S \Lambda C C \cdot 15$

$1 * \quad$ Vico (10-11-2011) 386*16.

349 (bb) + SVINTII. $\Lambda \cdot$ E

$+\mathrm{PIVS} \Lambda \mathrm{CCE}$

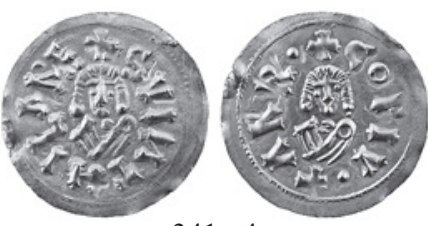

341g.4

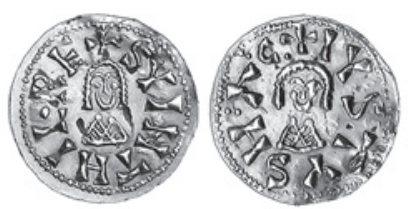

$347 b b .1$

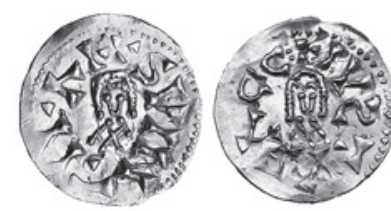

$348 \mathrm{dd} .1$

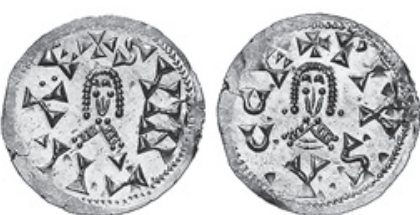

$349 \mathrm{bb} .1$

$51 *-53(1,34 ; 1,49 ; 1,43)$ Caballero, Maquedano \&

Sánchez 2010, 7*,8*, 10* (T. Vega Baja; M. Sta.

Cruz Toledo, 2005/53/972, 975, 977).

(b) + SVINTHIL $\Lambda$ RE ${ }^{X}$

+ TOLETOPIVs

14* (1,44) Caballero, Maquedano \& Sánchez 2010, 11* (T. Vega

Baja; M. Sta. Cruz Toledo, 2005/53/979).

(d) + SVINTHIL $\Lambda$ RE +

+ TOLETOPIV

17* (1,39) Caballero, Maquedano \& Sánchez 2010, 9* (T. Vega Baja; M. Sta. Cruz Toledo, 2005/53/976).

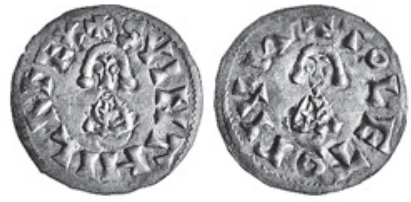

361 a.51

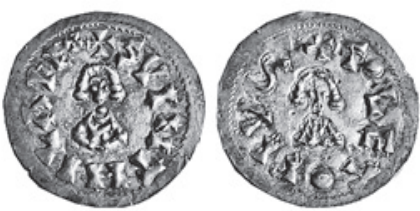

$361 \mathrm{~b} .14$

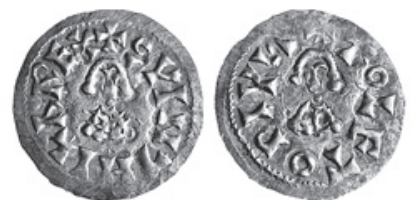

361d.17

15. Aunque parece que al final de la leyenda hay dos glóbulos, el superior es en realidad, muy simplificado, el brazo izquierdo de la cruz central.

16. Podría ser igual que las piezas de La Capilla recogidas bajo la entrada Pliego $348 \mathrm{~d}$, de la que únicamente se diferencia en que en ésta la primera $\mathrm{S}$ de la leyenda de reverso aparece en su dirección correcta -y no retrógrada como en la que presentamos aquí-, ya que en la obra de Fernández López (1895), por limitaciones propias de la época, no se detalla la dirección de las letras. 


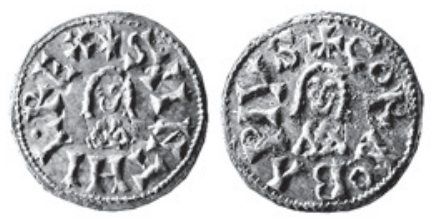

367a.19

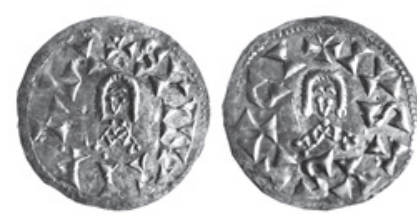

387 n. 10

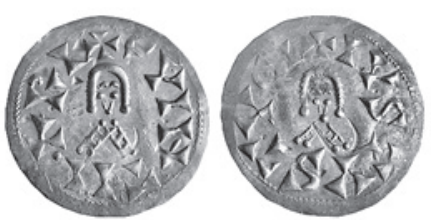

$387 \mathrm{p} .3$

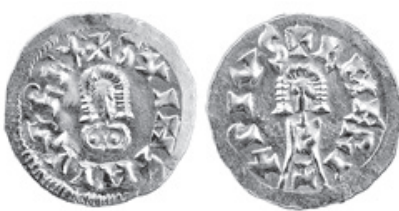

393a. 100

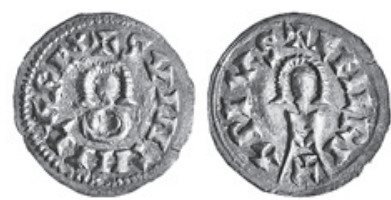

$393 b .61$

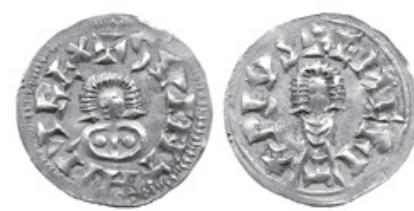

$393 b .64$

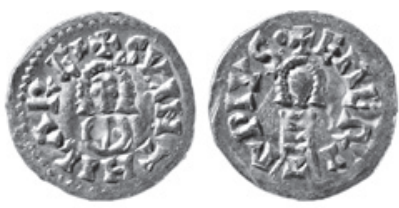

393d.13

Cordoba

367 (a) * SVINTIL $\bullet$ REX

$+\mathrm{COR} \Delta \mathrm{OB} \Lambda \mathrm{PIVS}$

19* (1,34) Valero, Arcos \& Molina 2011: 278* (H: La Vega Baja; M. Sta. Cruz Toledo).

Tvcci

387 (n) +SVINTII. $\Lambda$ RI

+ IVSTVSTVCI

10* (1,33) Caballero, Maquedano \& Sánchez 2010, 5* (T. Vega Baja; M. Sta. Cruz Toledo, 2005/53/973).

(p) + 2VINTII. $\Lambda$ RI

+ IVSTVSTVCI

$3^{*} \quad(1,33)$ Caballero, Maquedano \& Sánchez 2010, 6* (T. Vega Baja; M. Sta. Cruz Toledo, 2005/53/993).

Emerita

393 (a) +SVINTHIL $\Lambda$ REX

+ EMERI $|\mathrm{T}| \Lambda$ PIVS

79 (1,55) Vico (9-6-2005) 460* (M. Mérida, 37560)

95 (1,49) Durán (17-12-1997) 1369* (M. Mérida, 37062).

96 (1,45) Áureo (21-5-1998) 474* (M. Mérida, 37080).

97 (1,48) Áureo (22-9-1999) 326* (M. Mérida, 37126).

98 (1,45) Áureo (26-1-2000) 333* (M. Mérida, 37164).

99 (1,48) Áureo (16-12-2002) 448* (M. Mérida, 37418).

100* (1,45) Vico (5-6-2008) 499* (M. Mérida, 37688).

101 (1,52) Vico (13-11-2008) 442* (M. Mérida, 37747).

102 (1,48) Hervera (31-3-2009) 427* (M. Mérida, 37768).

(b) + SVINTHIL $\Lambda R^{X}$

+ EMERI $|\mathrm{T}| \Lambda$ PIVS

35 (1,47) Vico (9-6-2005) 459* (M. Mérida, 37559).

61* (1,32) Caballero, Maquedano \& Sánchez 2010, 3* (T. Vega Baja; M. Sta. Cruz Toledo, 2005/53/974).

62 (1,43) Áureo (16-12-1999) 2121* (M. Mérida, 37140).

63 (1,49) Áureo (1-3-2000) 1184* (M. Mérida, 37168).

64* (1,56) Áureo (4-5-2000) 148* (M. Mérida, 37201).

65 (1,50) Áureo (4-5-2000) 149* (M. Mérida, 37202).

66 (1,45) Vico (1-6-2000) 547* (M. Mérida, 37238).

67 Hervera (28-11-2000) 277* (M. Mérida, 37245).

68 (1,45) Vico (10-6-2004) 292* (M. Mérida, 37474).

69 (1,49) Hervera (10-7-2008) 505* (M. Mérida, 37697).

70 (1,49) Vico (5-6-2008) 500* (M. Mérida, 37687).

71 (1,50) Hervera (18-11-2008) 1421* (M. Mérida, 37740).

72 (1,47) Vico (13-11-2008) 443* (M. Mérida, 37748).

73 (1,48) Vico (2-4-2009) 1362* (M. Mérida, 37786). 
(d) + SVINTHIL $\Lambda$ RE $^{x}$

+ EMERI $|\mathrm{T}| \Lambda$ PIVS •

9 (1,44) Vico (9-6-2005) 461* (M. Mérida, 37561).

$13 * \quad(1,46)$ Hervera (18-11-2008) 1423* (M. Mérida, 37741).

(j) + SVINTHIL $\Lambda$ RE

+EMERI $|\mathrm{T}| \Lambda$ PIVS $\bullet$

11* (1,49) Hervera (10-12-2002) 894* (M. Mérida, 37396).

394 (b) +2VINTHIL $\Lambda$ RE

+EMERI $|\mathrm{T}| \Lambda$ PIVS

$11^{*}(1,49)$ Hervera (28-11-2000) 278* (M. Mérida, 37244).

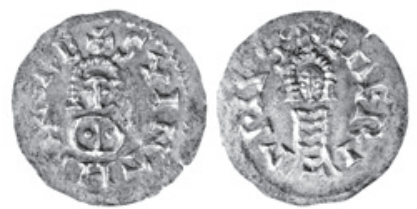

$393 \mathrm{j} .11$

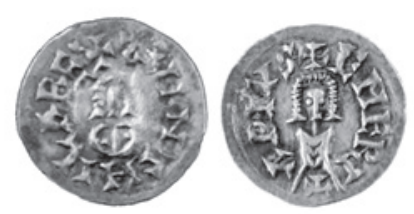

394b.11

Cepis

$412+$ SVINThIL $\Lambda R^{\mathrm{X}}$

+ CEPISPIVS

2* $\quad(1,49)$ Caballero, Maquedano \& Sánchez 2010, 4* (T. Vega Baja; M. Sta. Cruz Toledo, 2005/53/978).

Vada

431A +SVINTIL $\Lambda$ RE:

$+\mathrm{V} \Lambda \mathrm{D} \Lambda \mathrm{PIVS}$

$1^{*} \quad(1,40)$ Künker (26-09-2011) $1101^{*}=$ Pliego 2011, fig. 16*.

\section{SISENANDO}

Acci
438 (e) + SISIN $\Lambda \mathrm{I} \cdot \mathrm{VSRI}$
$+\operatorname{IVSTVS} \Lambda \mathrm{CI}$
2* $\quad(1,17)$ Caballero, Maquedano \& Sánchez 2010, 15* (T. Vega Baja; M. Sta. Cruz Toledo, 2005/53/981).
$(\mathrm{gg})+$ SISI:N $\Lambda \mathrm{N} \cdot I V S I:$
+ IVSTVS $\Lambda C I$
1* (1,11) Caballero, Maquedano \& Sánchez 2010, 16* (T. Vega Baja; M. Sta. Cruz Toledo, 2005/53/988).

\section{Castilona}

439
(c) $+\mathrm{SISE} \Lambda \Lambda И \cdot \mathrm{VSRE} \cdot$
$+\mathrm{C} \Lambda \mathrm{STEI} \Lambda \Lambda \mathrm{IVS}$
2* (1,02) Caballero, Maquedano \& Sánchez 2010, 17* (T. Vega Baja; M. Sta. Cruz Toledo, 2005/53/986).

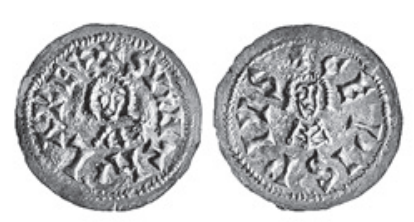

412.2

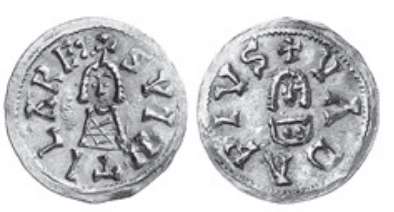

431A.1

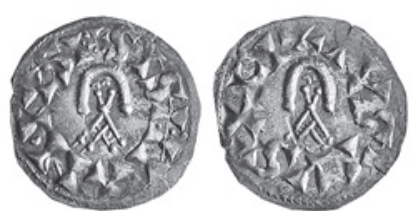

438e. 2

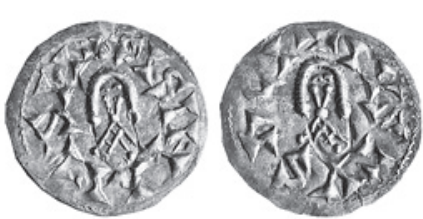

$438 \mathrm{gg}$

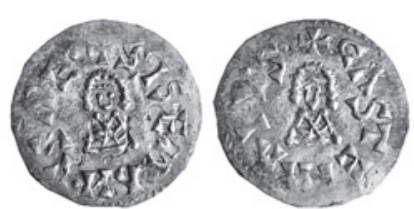

$439 c .2$ 


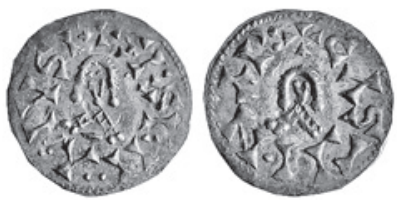

441f.2

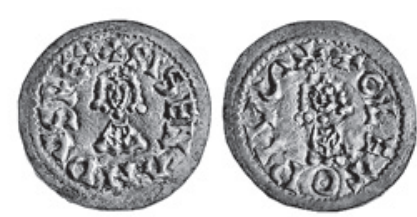

449a. 2

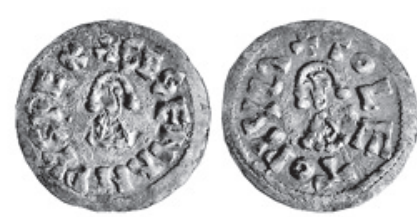

$449 b .5$

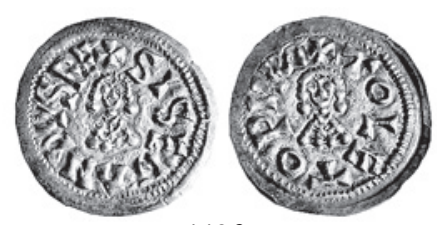

449f. 7

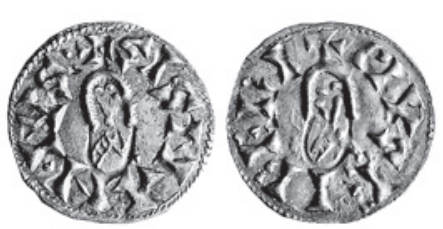

$458 \mathrm{~m} .1$

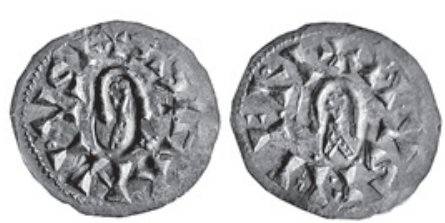

458n. 1

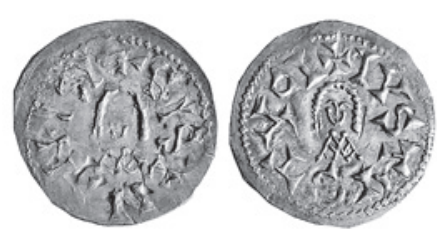

$462 \mathrm{k} .1$

441 (f) $+\operatorname{SESEN}^{*} \Lambda$. ...IVSR $^{17}$

$+\mathrm{C} \Lambda \mathrm{STII} \cdot \mathrm{N} \Lambda \mathrm{P}$ :

$2^{*} \quad(1,26)$ Caballero, Maquedano \& Sánchez 2010, 18* (T. Vega Baja; M. Sta. Cruz Toledo, 2005/53/990).

Toleto

449 (a) + SISEN $\Lambda$ NDVSREX

+ TOLETOPIV

2* (1,35) Caballero, Maquedano \& Sánchez 2010, 12* (T. Vega Baja; M. Sta. Cruz Toledo, 2005/53/983).

(b) + SESEN $\Lambda$ NPVSREX

+ TOLETOPIVu

5* $\quad(1,53)$ Caballero, Maquedano \& Sánchez 2010, 14* (T. Vega Baja; M. Sta. Cruz Toledo, 2005/53/987).

(f) + SISEN $\Lambda$ NPVSRE

+ TOLETOPIVU

7* $\quad(1,50)$ Caballero, Maquedano \& Sánchez 2010, 13* (T. Vega Baja; M. Sta. Cruz Toledo, 2005/53/985).

Eliberri

$458(\mathrm{~m})+\mathrm{SISIN} \Lambda \mathrm{NI}{ }^{\circ} \mathrm{VSR}$

+ PIVSEII:ERI

1* $\quad(1,29)$ Caballero, Maquedano \& Sánchez 2010, 20* (T. Vega Baja; M. Sta. Cruz Toledo, 2005/53/982) ${ }^{18}$.

(n) + SISN $\Lambda \mathrm{N} \bullet I V S \bullet$

+ PIVSEIBERI

1* $\quad(1,26)$ Caballero, Maquedano \& Sánchez 2010, 21* (T. Vega Baja; M. Sta. Cruz Toledo, 2005/53/984).

Tvcci

$462(\mathrm{k})+$ SISEN $\Lambda$ NqSR. ${ }^{19}$

+ IVSTVSTVCI

1* $\quad(1,14)$ Caballero, Maquedano \& Sánchez 2010, 23* (T. Vega Baja; M. Sta. Cruz Toledo, 2005/53/991).

(1) + SISEH $\Lambda \mathrm{HA}^{*}: \mathrm{SR}^{\bullet 20}$ + IVSTVSTCI

1* (1,21) Caballero, Maquedano \& Sánchez 2010, 22* (T. Vega Baja; M. Sta. Cruz Toledo, 2005/53/980).

17. El brazo izquierdo de la cruz central completa la $\mathrm{R}$ final de la leyenda de anverso.

18. Debido a que añadimos dos nuevas variantes ( $m$ y $n$ ) a nuestra entrada $n^{\circ} 258$, nuestra antigua (m) pasaría a ser (o), cambio que no implica ningún problema de clasificación ya que bajo la misma se recogen 14 monedas conocidas a través de referencias antiguas aunque sus leyendas son inciertas.

19. Parece que el brazo izquierdo de la cruz central completa la $\mathrm{R}$ final de la leyenda de anverso.

20. Parece que el brazo izquierdo de la cruz central completa la $\mathrm{R}$ final de la leyenda de anverso. 
Emerita

467 (h) + SISEN $\Lambda$ NDVSRE ${ }^{\mathrm{X}}$

+ EMERI $|\mathrm{T}| \Lambda$ PIVS

20 (1,48) Vico (16-11-2006) 530* (M. Mérida, 37611).

Bracara

a/ 2 a cada lado del tipo.

469 (b) +2IटЕИ ИИРRE•

$+\mathrm{BR} \Lambda \mathrm{C} \Lambda \cdot \Lambda \mathrm{I}^{\bullet} \mathrm{IV} \bullet$

1* (1,31) Caballero, Maquedano \& Sánchez 2010, 19* (T. Vega Baja; M. Sta. Cruz Toledo, 2005/53/989).

\section{CHINTILA}

Castilona 478

+ CHINTIL $\Lambda$ R

+ C $\Lambda$ STEI.ON $\Lambda$ PIVS

2* $(1,07)$ Pliego \& Urgorri, 2*.

Mentesa

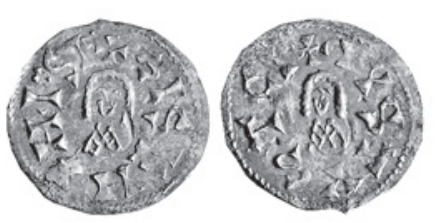

4621.1

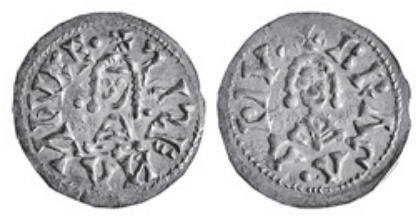

$469 \mathrm{~b} .1$

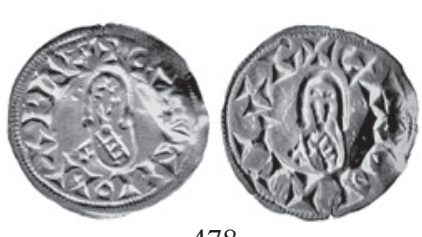

478

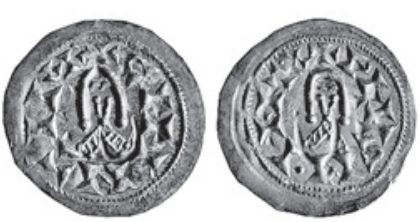

481A.1

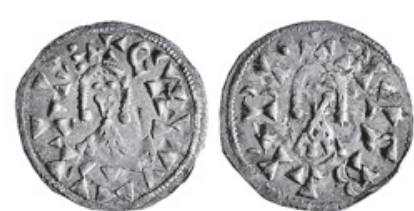

485a.1

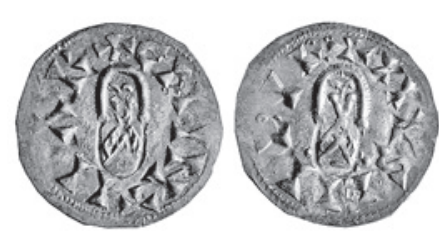

491c. 1

491 (c) + CNINTI I $\Lambda \mathrm{R}$

+ IIVSEIIBIR:

1* (1,21) Caballero, Maquedano \& Sánchez 2010, 27* (T. Vega Baja; M. Sta. Cruz Toledo, 2005/53/1000). 


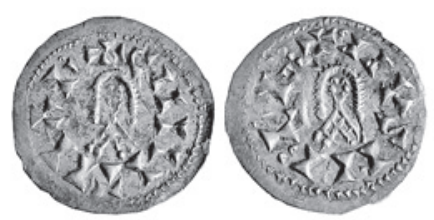

491d.1

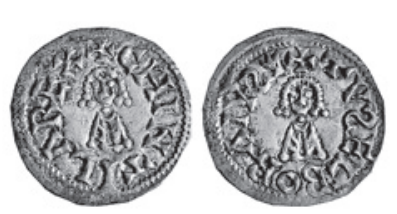

496A.1

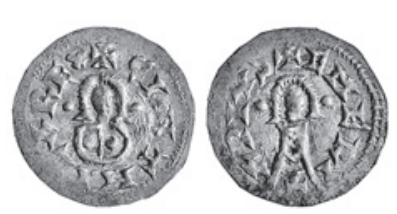

499a.5

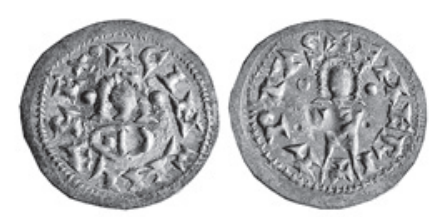

499d.5

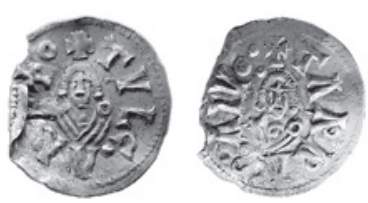

$509 \mathrm{~b} .1$

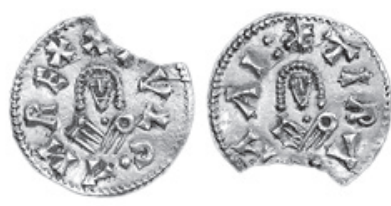

510a.1

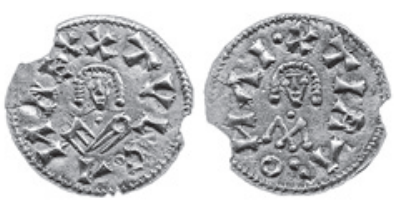

$510 \mathrm{~b} .1$ (d) + C II I II TI I $\Lambda \mathrm{R}$

+ TIVSLI.I :LR:

1* (1,14) Caballero, Maquedano \& Sánchez 2010, 26* (T. Vega Baja; M. Sta. Cruz Toledo, 2005/53/998).

Elbora

496A

+ CHINTIL $\Lambda$ REX

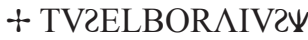

1* $\quad(1,52)$ Caballero, Maquedano \& Sánchez 2010, 28* (T. Vega Baja; M. Sta. Cruz Toledo, 2005/53/996).

Emerita

498

+ CHINTHIL $\Lambda R^{X}$

+ EMERE $|\mathrm{T}| \Lambda$ PIVS

1* (1,39) Vico (9-6-2005) 463* = CNV 383.1 (M. Mérida, 37562).

499 (a) + CINTHIL $\Lambda$ REx

+ EMERI $|\mathrm{T}| \Lambda$ PIVS

5* $\quad(1,42)$ Caballero, Maquedano \& Sánchez 2010, 31* (T. Vega Baja; M. Sta. Cruz Toledo, 2005/53/1001).

(d) + CINTNIL $\Lambda$ REx $\bullet$

+ EMERI $|\mathrm{T}| \Lambda$ PIVS

5*-6 (1,37; 1,51) Caballero, Maquedano \& Sánchez 2010, 29*-30* (T. Vega Baja; M. Sta. Cruz Toledo, 2005/53/995, 997).

\section{TULGA}

Tarracona

509

(b) $+\mathrm{TVLC} \cdot \Lambda \mathrm{RE}+\odot$

$+\mathrm{T} \Lambda \mathrm{RR} \Lambda \mathrm{CO}: \Lambda \mathrm{IV} . \mathrm{O}:$

1* $\quad(1,3)$ De Juan, Gallego \& García 2009: 131* (H: Vega Baja; M. Sta. Cruz Toledo, 6547).

Tirasona

510 (a) +TVLC $\bullet \Lambda$ REX

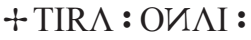

$1^{*} \quad(1,18)$ Amardel 1896 = Barral: 186, 107 = Richard 2005, 53* (H: Narbona; M. Narbonne) ${ }^{21}$.

(b) +TVLC $\cdot \Lambda$ REX

$+\mathrm{TIR} \Lambda: \mathrm{O} \Lambda \Lambda \mathrm{I}^{\bullet}$

$1^{*} \quad$ Archivo J. Herrero, S.A.

21. Ex Berthomieu. 


\section{CHINDASVINTO}
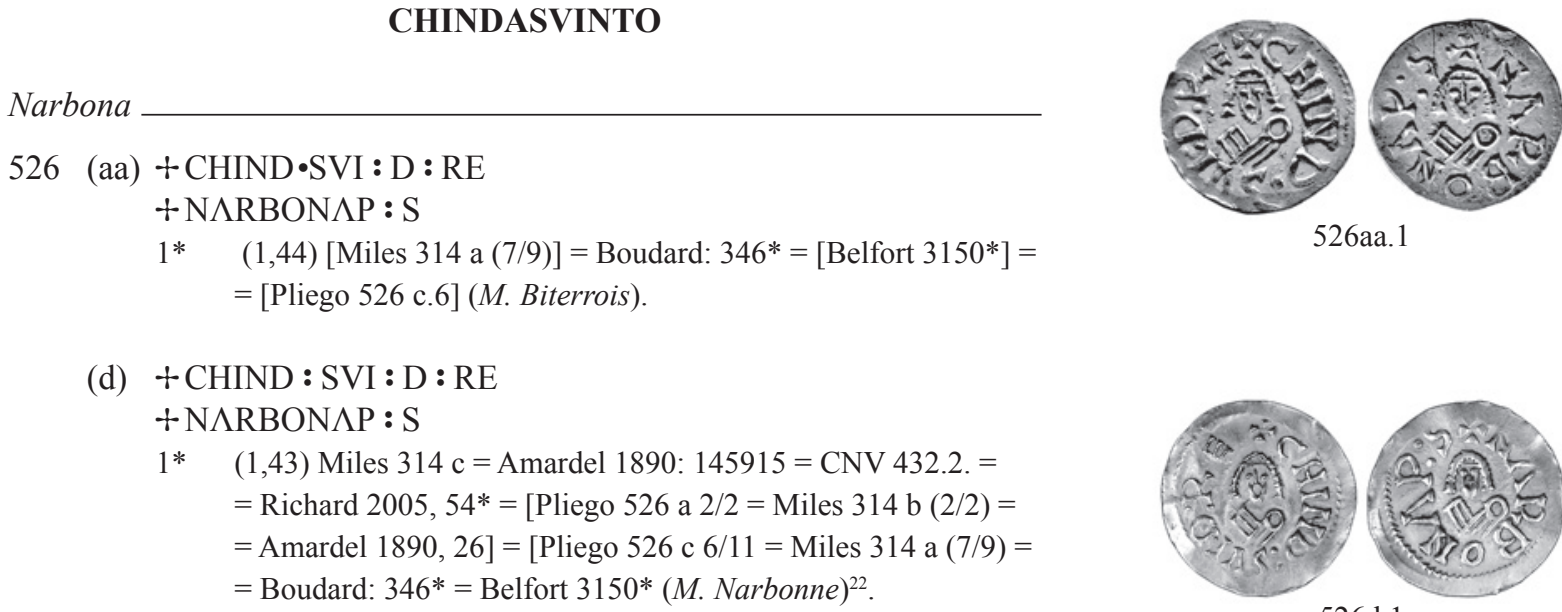

526aa. 1

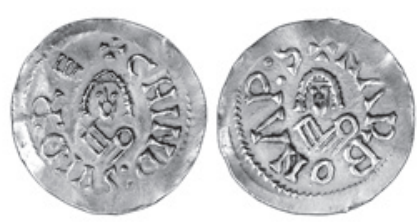

$526 \mathrm{~d} .1$

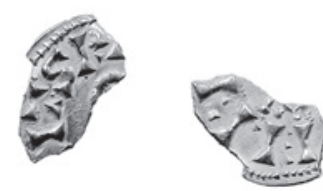

$542 b .1$

a/ + a cada lado del busto de anverso

r/, a cada lado del busto de reverso.

550 (gg) + CASVINOVSP4

+EMER | I | T $\Lambda$ PIVS

1* Vico (3-3-2011) 447*.

\section{RECESVINTO}

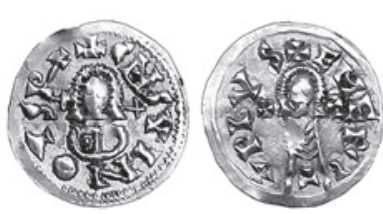

550gg.1

Toleto

580 (c) +RECCES IṾI INQVSP4

+ TOLETOPIVS

$12^{*}(1,52)$ (H: Calahorra).

\section{Emerita}

$604+$ RECCES I V I INOVSP

+ EMERITAPIVS

17* Vico (4-6-2009) 362* (M. Mérida ${ }^{23}$ ).

22. Ex Musée de Béziers.

23. Aún no ha sido asignado número de inventario.

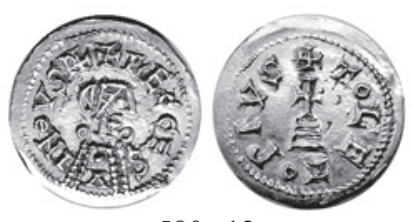

580c. 12

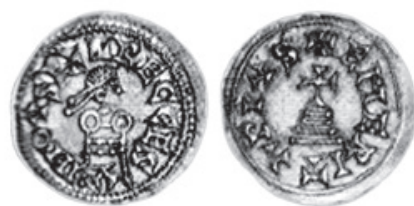

604.17
ISSN: 1133-4525 ISSN-e: 2255-3924

http://dx.doi.org/10.12795/spal.2012.i21.12 


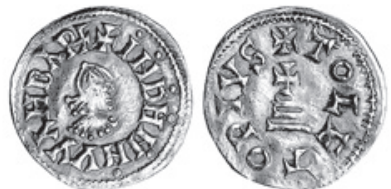

$617 b .1$

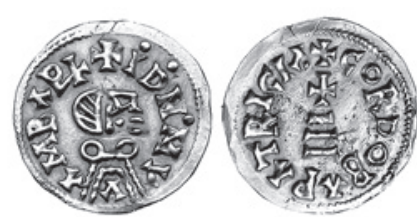

618A.1

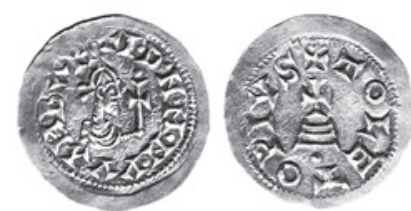

$618 \mathrm{e} .38$

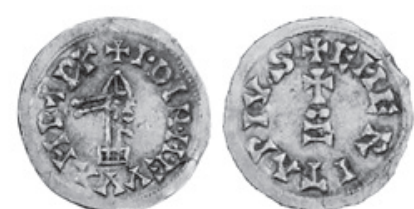

628a.7

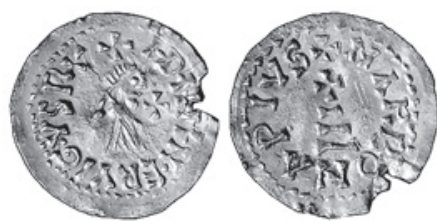

$631 b .7$

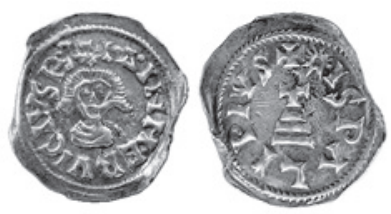

$649 \mathrm{f} .7$

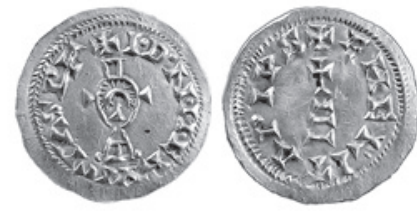

$658 f .3$

WAMBA

Toleto

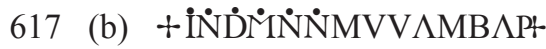
+ TOLETOPIVS

$1 * \quad$ Vico (1-3-2012) 384*.

618 (e) $+\mathrm{I} \cdot \mathrm{D}^{\bullet} \mathrm{N}^{\bullet} \mathrm{M}^{\bullet} \mathrm{N}{ }^{\bullet} \mathrm{VV} \Lambda \mathrm{MB} \Lambda \mathrm{P} 4$

+ TOLETOPIVS

38* (1,5) (H: Plaza de Cambil, n² 2, Jaén; M. Jaén DJ/NU06625).

Cordoba

618A + + İD ${ }^{\bullet} \mathrm{MVIVI} \Lambda \mathrm{MB} \Lambda \mathrm{P} 4$

$+\mathrm{CORDOB} \Lambda \mathrm{P} \Lambda \mathrm{TRICI} \Lambda$

$1^{*} \quad$ Vico (1-3-2012) 383*.

Emerita

628 (a) $+\mathrm{I}^{\bullet} \mathrm{D}^{\bullet} \mathrm{IN} \mathrm{M}^{\bullet} \mathrm{EVV} \Lambda \mathrm{MB} \Lambda \mathrm{P} 4$

+ EMERIT $\Lambda$ PIVS

7* $\quad(1,45)$ Áureo (1-3-2000) 1187* (M. Mérida, 37170).

\section{ERVIGIO}

Narbona

631 (b) $+\mathrm{I} \cdot \mathrm{D} \cdot \mathrm{N} \cdot \mathrm{M} \cdot \mathrm{N} \cdot \mathrm{ERVIG} \cdot \mathrm{VSP} 4$ $+\mathrm{N} \Lambda \mathrm{RBON} \Lambda \mathrm{PIVS}$

7* $(1,42)$ Miles 396 a $(7 / 8)=$ Amardel 1890, $3=$ Richard 2005, 55* (H: Narbona; M. Narbonne).

Ispali

649 (f) $+\mathrm{I}^{\bullet} \Delta^{\bullet} \mathrm{INM}^{\bullet} \mathrm{ERVICIVSP}-$

+ ISP $\Lambda$ LIPIVS

7* $\quad(1,5)$ De Juan, Gallego \& García 2009: 131* (H: Vega Baja; M. Sta. Cruz Toledo, 7086).

Emerita

658 (f) $+\mathrm{I} \cdot \mathrm{D} \bullet \mathrm{N} \cdot \mathrm{M} \cdot \mathrm{ERVIGIVSP} 4$ + EMERIT $\Lambda$ PIVS

3* $(1,45)$ Herrero (28-5-2008) 201* = [Pliego 658 d.7] (M. Mérida, 37720). 
EGICA

Narbona

662 (c) $+\mathrm{I} \cdot \mathrm{D} \cdot \mathrm{N} \cdot \mathrm{M} \cdot \mathrm{N} \cdot \mathrm{EGIC} \Lambda \mathrm{P} 4$ $+\mathrm{N} \Lambda \mathrm{RBON} \Lambda \mathrm{PIVS}$

$3^{*} \quad(1,48)$ Miles 419 b (3/6) = Boudard: $347=$ Amardel 1890, $4=$ $=$ Belfort $3166^{*}=$ Barral: $192,148^{*}=$ Richard 2005, 56* $(\mathrm{H}$ : Lézignan-la-Cèbe, Hérault; M. Narbonne).

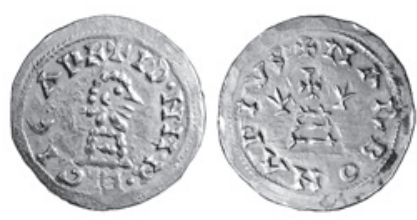

$662 \mathrm{c} .3$

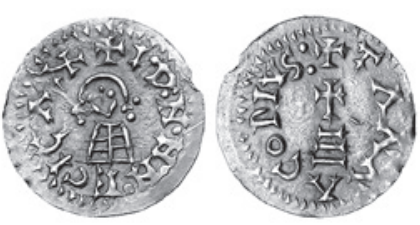

671AA.1

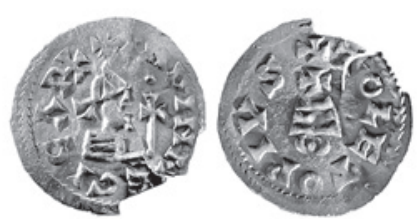

$682 \mathrm{c} .1$

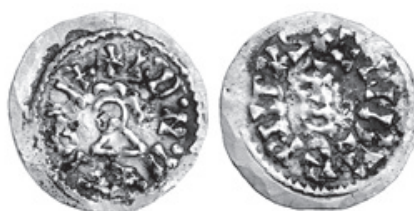

693a.1

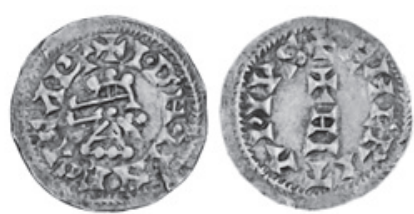

$703 b .11$

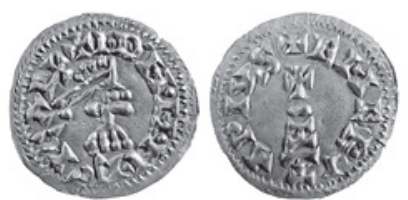

705.7

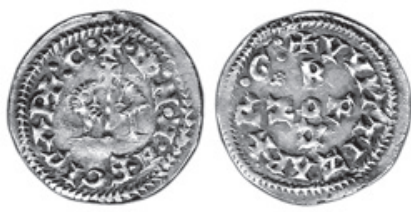

$716 \mathrm{cc} .1$ 


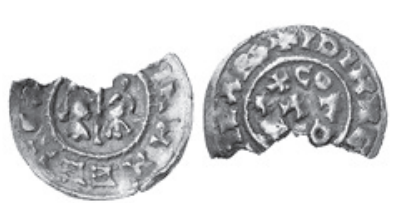

733d. 1

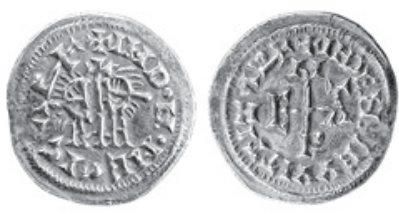

$753 \mathrm{a} .2$

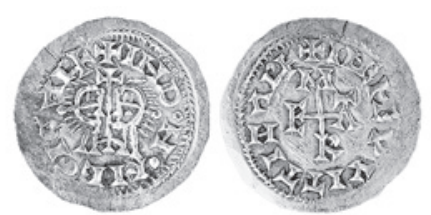

$753 \mathrm{~b} .4$

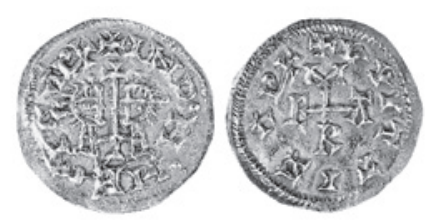

7530.9

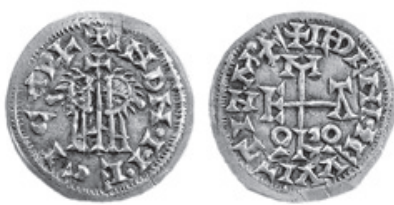

753v. 2

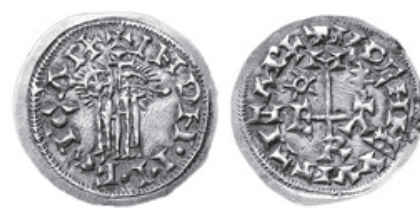

753 cc. 5
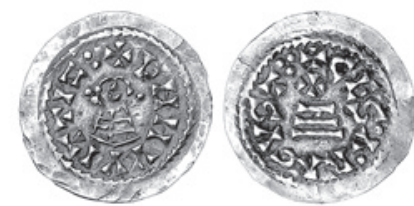

76211. 1

Cordoba

a 2 a ambos lados del cetro

733

(d) + [IND]INMNEEGIC $\Lambda[\mathrm{P}$ - $]$

+ IDINME[VVITT]IS $\Lambda$ P4

$1^{*} \quad(1,02)$ Áureo (29-04-2010) 2393*. Faltada.

Emerita

753

(a) $+\mathrm{IND}^{\bullet} \mathrm{N} \bullet \mathrm{MEGIC} \Lambda \mathrm{P} 4$

+ INDENMEVVITTIS $\Lambda$ P

2* $\quad(1,46)$ Herrero (28-5-2008) 205* (M. Mérida, 37721).

3 (0,95) Áureo (16-12-2002) 453* (M. Mérida, 37419).

(b) $+\mathrm{IND}^{\bullet} \mathrm{N}^{\bullet} \mathrm{MEGIC} \Lambda \mathrm{P} 4$

+ INEMEVVITTIS $\Lambda$ P

4* $\quad(1,44)$ Hervera (18-11-2008) 1425* (M. Mérida, 37742).

(o) $+\mathrm{INDN}^{\bullet} \mathrm{M}^{\bullet} \mathrm{EGIC} \Lambda \mathrm{P} 4$

+ VVITTIS $\Lambda \mathrm{P} 4$

9* (1,45) Vico (13-11-2008) 445* (M. Mérida, 37749).

(v) $+\mathrm{INDN}^{\bullet} \mathrm{M}^{\bullet} \mathrm{EGIC} \Lambda \mathrm{P} 4$ + INDENMEVVITTZ $\Lambda$ P

2* (1,41) Vico (5-6-2008) 508* (M. Mérida, 37689).

(cc) $+\mathrm{INDN}^{\bullet} \mathrm{M}^{\bullet} \mathrm{EGIC} \Lambda \mathrm{P} 4$

+ INDENMEVVITTIZ $\Lambda$ P

5* $(1,52)$ (Fondo Antiguo) (M. Mérida, 28944).

\section{WITIZA}

Cesaragusta

762 (11) +DNUVVITTIS:

$+\mathrm{CES}^{\bullet} \Lambda \cdot \mathrm{R} \Lambda \mathrm{CVST}^{*}:$

1* Vico (10-11-2011) 393*.

Toleto

A Tipo 14 c. ¿ý sobre el cetro?

$\mathrm{R}$ Cruz sobre 3 peldaños.

$771 \mathrm{~A}+[\ldots \mathrm{VV}] \mathrm{ITIZ} \Lambda \mathrm{P} 4$

+ TOLE[TOPI]VS

$1^{*} \quad(0,80)$ Herrero (15-12-2011) $397^{*}$. 


\section{RODERICO}

Egitania

794 (c) + INDNERVPERICVSP4

+ EGIT $\Lambda$ NI $\Lambda$ PIVS

$3^{*} \quad(1,49)[$ Miles $512 \mathrm{c}(2 / 7)]=$ Ex Ferreira $77=$ Miles $1966,72 *=$ = Pliego 2010: 97, fig. 3.1* (HSA 1001.57.617).

\section{AGILA II}

Narbona

795 (b) $+\mathrm{I}^{\bullet} \mathrm{d}{ }^{\circ} \mathrm{N} \Lambda$ :CHIL $\Lambda \mathrm{P} 4$

+ + $\Lambda$ RBON $\Lambda$ PIV2

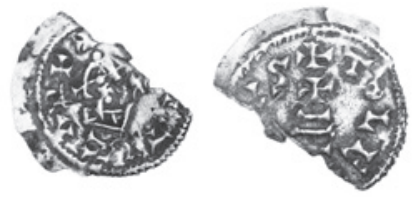

771A.1

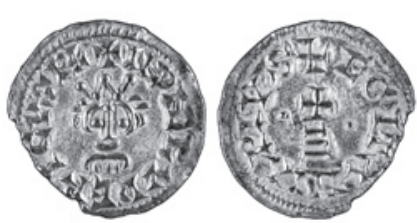

$794 c .3$

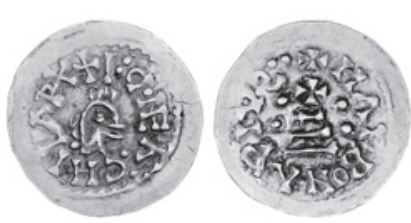

795b.1

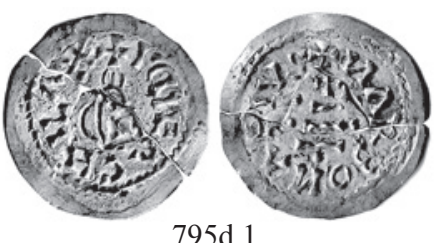

$795 \mathrm{~d} .1$

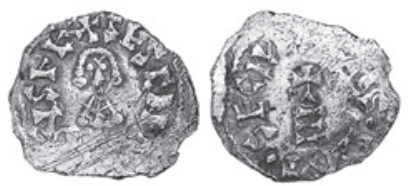

814A.1

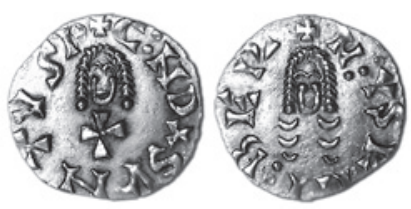

1136A.1
FeCHA DE ENTRADA: 05/03/2012

FECHA DE ACEPTACIÓN: 19/03/2012 